\title{
Potential Theory of Moderate Markov Dual Processes
}

\author{
P. J. Fitzsimmons • R. K. Getoor
}

Received: 14 November 2008 / Accepted: 13 April 2009 / Published online: 22 May 2009

(C) The Author(s) 2009. This article is published with open access at Springerlink.com

\begin{abstract}
Let $X$ be a Borel right Markov process, let $m$ be an excessive measure for $X$, and let $\widehat{X}$ be the moderate Markov dual process associated with $X$ and $m$. The potential theory of co-excessive measures (i.e., measures that are excessive for $\widehat{X}$ ) is developed with special emphasis on the Riesz decomposition. This is then applied to obtain the Riesz decomposition of excessive functions (of $X$ ) by exploiting the correspondence between such functions and co-excessive measures. The potential theory of co-excessive measures also enables us to discuss Walsh's interior réduite under minimal conditions. Many of the tools of the theory of Markov processes are employed in this development. For example, Kuznetsov measures, Ray compactifications, $h$-transforms, and duality theory for Borel right processes.
\end{abstract}

Keywords Potential theory • Excessive measure $\cdot$ Kuznetsov measure • Duality • $h$-transform $\cdot$ Riesz decomposition $\cdot$ Réduite $\cdot$ Balayage

Mathematics Subject Classifications (2000) Primary 60J40 • Secondary 60J45

\section{Introduction}

The potential theory of the excessive measures of a Borel right Markov process, with a distinguished excessive measure $m$ serving as background measure, has been well understood since the 1986 paper [8] of Bernard Maisonneuve and the first-named author of this paper. An expository account appears in [13]. Slightly earlier, Joe Glover and the second-named author treated in [15] some aspects of the general case and obtained complete results under a weak duality hypothesis. The potential theory of the excessive functions of a general Borel right Markov process has not been treated as systematically heretofore. Of course, under weak duality there is a

P. J. Fitzsimmons · R. K. Getoor $(\bowtie)$

University of California, San Diego, San Diego, CA, USA

e-mail: rgetoor@ucsd.edu 
one-to-one correspondence between excessive functions and co-excessive measures, and in [15] this correspondence was exploited to treat the Riesz decomposition of excessive functions. For a general Borel right process $X$ with distinguished excessive measure $m$ there is always a left continuous moderate Markov dual process $\widehat{X}$, and there is a one-to-one correspondence between excessive functions of $X$ and coexcessive measures (i.e., excessive measures for $\widehat{X}$ ). Thus to study excessive functions of $X$ it is natural to develop the potential theory of the excessive measures of a leftcontinuous moderate Markov process. This study is carried out in the present paper when the moderate Markov process is the dual of a Borel right process $X$. At the end of Section 7, this is applied to obtain results about the excessive functions of $X$.

As one might expect, the theory is quite similar to that for excessive measures. But there are important differences, and certain technical difficulties. As for excessive measures, one of the main tools is the Kuznetsov measure $\mathbf{Q}_{m}$ governing the Kuznetsov process $Y=\left(Y_{t}\right)_{t \in \mathbf{R}}$ associated with $X$ and $m$. In fact, $\widehat{X}$ is most conveniently defined in terms of $Y$. A key tool in the present theory, which did not arise in the study of excessive measures, is the notion of $h$-transforms for $X$. This is not surprising since such transforms play a key role in [15] and in Chapter 13 on duality in [3]. If $h$ is an excessive function (of $X$ ) that is finite $m$-a.e., then $\xi:=h \cdot m$ is a co-excessive measure. The class of co-excessive measures that can be so represented coincides with the class of those that are absolutely continuous with respect to $m$. In the remainder of this introductory section the co-excessive measure $\xi$ will be taken from this class, with representation $\xi=h \cdot m, h$ being excessive.

In analogy with excessive measures, the Kuznetsov measure $\widehat{\mathbf{Q}}_{\xi}$ associated with $\widehat{X}$ and the co-excessive measure $\xi$ will be a key tool. But it is well known that $\xi$ is excessive for $X^{h}$ (the $h$-transform of $X$ ) and $\widehat{\mathbf{Q}}_{\xi}$ is just the time reversal of $\mathbf{Q}_{m}^{h}$, the Kuznetsov measure associated with $X^{h}$ and $\xi$. It turns out to be more convenient to use $\mathbf{Q}_{m}^{h}$ rather than $\widehat{\mathbf{Q}}_{\xi}$ in most instances; this notation emphasizes the excessive function $h$, which is the eventual object of our development. We shall also make use of the Ray compactifications of $X$ and $\widehat{X}$. For $X$ this is a standard tool in the theory of Markov processes. In the case of a moderate Markov processes this material is not so well known, but it receives an excellent treatment in [3].

Precise definitions are given in Section 2, where the basic properties of the Kuznetsov process are reviewed. The Ray-Knight compactification of $X$ is also summarized there. Section 3 introduces the moderate Markov dual process $\widehat{X}$. For the most part this section is also a review. The elementary properties of $h$-transforms and their relationship with the Kuznetsov measure $\mathbf{Q}_{m}^{h}$ are recorded at the beginning of Section 4. Proposition 4.1 extends to $\mathbf{Q}_{m}^{h}$ a standard property of $h$-transforms. Theorem 4.4 is the key technical fact that makes $\mathbf{Q}_{m}^{h}$ a useful tool for the remainder of the paper. The Ray-Knight compactification of $\widehat{X}$ is contained in Section 5. Here we follow [3], although it necessary to adapt their hypotheses to our situation. We prove the key separation result of Walsh under our hypotheses in Theorem 5.2. In Section 6 we come to one of the places where the potential theory of co-excessive measures differs from that of excessive measures. Namely, we are unable to prove that a purely excessive measure may be written uniquely as the integral of an entrance law. However, by relaxing the customary $\sigma$-finiteness requirement in the definition of the notion "entrance law", we do obtain a unique representation in terms of what we call "loose" entrance laws. This is recorded as Theorem 6.3. It is somewhat surprising that one can prove the uniqueness of the representation without assuming 
that the measures involved are $\sigma$-finite. This allows us in Theorem 6.6 to extend the moderate Markov property to the death time $\beta$ of $Y$ in a manner analogous to the familiar extension of the strong Markov property to the birth time $\alpha$ of $Y$. See, for example, $[13,(6.15)]$. Our proof is patterned on that used for the extended strong Markov property in [8]. The results of the preceding sections are used in Section 7 to pursue our original objective of obtaining potential theoretic results for co-excessive measures (Theorem 7.8) and for excessive functions (Corollary 7.9).

Walsh has defined the "interior réduite" of an excessive function $h$ on a set $A$, denoted by $p_{A} h$, and has obtained various properties and characterizations of $p_{A} h$ under strong duality hypotheses and a strong Feller condition on the dual process. In Section $8, p_{A} h$ is investigated in the framework of this paper. It turns out that the co-excessive measure $p_{A} h \cdot m$ is a co-balayage of the co-excessive measure $\xi=h \cdot m$, similar to Hunt's balayage for excessive measures, as re-interpreted in [8]. See also [13]. Theorems 8.7 and 8.9 are direct generalizations under our minimal hypotheses of results found in Section 13.12 of [3]. It is also observed in Section 8 that the cone of co-potential measures is not a solid subcone of the cone of co-excessive measures in general. Thus a basic property of excessive measures fails for co-excessive measures.

We close this introduction with a few words on notation. We shall use $\mathcal{B}$ to denote the Borel subsets of the real line $\mathbf{R}$. If $(F, \mathcal{F}, \mu)$ is a measure space, then $b \mathcal{F}$ (resp. $p \mathcal{F}$ ) denotes the class of bounded real-valued (resp. $[0, \infty]$-valued) $\mathcal{F}$-measurable functions on $F$. For $f \in p \mathcal{F}$ we shall use $\mu(f)$ to denote the integral $\int_{F} f d \mu$; similarly, if $D \in \mathcal{F}$ then $\mu(f ; D)$ denotes $\int_{D} f d \mu$. We write $\mathcal{F}^{*}$ for the universal completion of $\mathcal{F}$; that is, $\mathcal{F}^{*}=\cap_{v} \mathcal{F}^{v}$, where $\mathcal{F}^{v}$ is the $v$-completion of $\mathcal{F}$ and the intersection runs over all finite measures on $(F, \mathcal{F})$. If $(E, \mathcal{E})$ is a second measurable space and $K=K(x, d y)$ is a kernel from $(F, \mathcal{F})$ to $(E, \mathcal{E})$ (i.e., $F \ni x \mapsto K(x, A)$ is $\mathcal{F}$-measurable for each $A \in \mathcal{E}$ and $K(x, \cdot)$ is a measure on $(E, \mathcal{E})$ for each $x \in F)$, then we write $\mu K$ for the measure $A \mapsto \int_{F} \mu(d x) K(x, A)$ and $K f$ for the function $x \mapsto \int_{E} K(x, d y) f(y)$.

\section{Preliminaries}

Throughout this paper $\left(P_{t}: t \geq 0\right)$ will denote a Borel right semigroup on a Lusin state space $(E, \mathcal{E})$, and $X=\left(X_{t}, \mathbf{P}^{x}\right)$ will denote a right-continuous strong Markov process realizing $\left(P_{t}\right)$. We shall specify the realization shortly. Recall that a (positive) measure $m$ on $(E, \mathcal{E})$ is excessive provided $m P_{t} \leq m$ for all $t \geq 0$. Since $\left(P_{t}\right)$ is a right semigroup, it follows that $m P_{t} \uparrow m$ setwise as $t \downarrow 0$. See [6, XII.36-37]. Let Exc denote the cone of excessive measures. In general, we shall use the standard notation for Markov processes without special mention. See, for example, [1, 6, 19], and [13]. In particular, $U^{q}:=\int_{0}^{\infty} e^{-q t} P_{t} d t, q \geq 0$, denotes the resolvent of $\left(P_{t}\right)$.

We are going to need the Kuznetsov process (or measure) $\mathbf{Q}_{m}$ associated with $\left(P_{t}\right)$ and a given $m \in$ Exc. We refer the reader to Section 6 of [13] for notation and definitions. See also [7]. For the convenience of the reader we shall review some of the basic notation here. Thus $W$ denotes the space of all paths $w: \mathbf{R} \rightarrow E_{\Delta}:=$ $E \cup\{\Delta\}$ that are right continuous and $E$-valued on an open interval $] \alpha(w), \beta(w)[$ and take the value $\Delta$ outside of this interval. Here $\Delta$ is a point adjoined to $E$ as an isolated point. The dead path $[\Delta]$, constantly equal to $\Delta$, corresponds to the interval being empty; by convention $\alpha([\Delta])=+\infty, \beta([\Delta])=-\infty$. The $\sigma$-algebra $\mathcal{G}^{\circ}$ on $W$ is 
generated by the coordinate maps $Y_{t}(w)=w(t), t \in \mathbf{R}$, and $\mathcal{G}_{t}^{\circ}:=\sigma\left(Y_{s}: s \leq t\right)$. Two families of shift operators are defined on $W$ : the simple shifts $\sigma_{t}, t \in \mathbf{R}$,

$$
\sigma_{t} w(s)=\left[\sigma_{t} w\right](s):=w(t+s), \quad s \in \mathbf{R},
$$

and the truncated shifts $\theta_{t}, t \in \mathbf{R}$,

$$
\theta_{t} w(s)=\left[\theta_{t} w\right](s):= \begin{cases}w(t+s), & s>0 \\ \Delta, & s \leq 0 .\end{cases}
$$

(In [9], the truncated shift operator was denoted $\tau_{t}$; here we follow [13] in using $\theta_{t}$.) Given $m \in$ Exc, the Kuznetsov measure $\mathbf{Q}_{m}$ is the unique $\sigma$-finite measure on $\mathcal{G}^{\circ}$, not charging $\{[\Delta]\}$, such that, for $-\infty<t_{1}<t_{2}<\cdots<t_{n}<+\infty$,

$$
\begin{aligned}
& \mathbf{Q}_{m}\left[Y_{t_{1}} \in d x_{1}, Y_{t_{2}} \in d x_{2}, \ldots, Y_{t_{n}} \in d x_{n}\right] \\
& \quad=m\left(d x_{1}\right) P_{t_{2}-t_{1}}\left(x_{1}, d x_{2}\right) \ldots P_{t_{n}-t_{n-1}}\left(x_{n-1}, d x_{n}\right) .
\end{aligned}
$$

Because of its crucial role in our development we recall the modified process $Y^{*}$ of $[13,(6.12)]$. Let $d$ be a totally bounded metric on $E$ compatible with the topology of $E$, and let $\mathcal{D}$ be a countable uniformly dense subset of the $d$-uniformly continuous bounded real-valued functions on $E$. Given a strictly positive $b \in b \mathcal{E}$ with $m(b)<\infty$ define $W(b) \subset W$ by the conditions:

(i) $\alpha \in \mathbf{R}$,

(ii) $Y_{\alpha+}:=\lim _{t \downarrow \alpha} Y_{t}$ exists in $E$,

(iii) $U^{q} g\left(Y_{\alpha+1 / n}\right) \rightarrow U^{q} g\left(Y_{\alpha+}\right)$ as $n \rightarrow \infty$, for all $g \in \mathcal{D}$ and all rationals $q>0$,

(iv) $U b\left(Y_{\alpha+1 / n}\right) \rightarrow U b\left(Y_{\alpha+}\right)$ as $n \rightarrow \infty$.

Evidently $\sigma_{t}^{-1}(W(b))=W(b)$ for all $t \in \mathbf{R}$, and $W(b) \in \mathcal{G}_{\alpha+}^{0}$ since $E$ is a Lusin space. We now define

$$
Y_{t}^{*}(w)= \begin{cases}Y_{\alpha+}(w), & \text { if } t=\alpha(w) \text { and } w \in W(b), \\ Y_{t}(w), & \text { otherwise. }\end{cases}
$$

Fix $m \in$ Exc and $b$ as above. If $m=\eta+\pi=\eta+\rho U$ is the Riesz decomposition [13, (5.33),(6.19)] of $m$ into harmonic and potential parts, then $\mathbf{Q}_{m}=\mathbf{Q}_{\eta}+\mathbf{Q}_{\pi}$, $\mathbf{Q}_{\eta}(W(b))=0$, and $\mathbf{Q}_{m}(\cdot ; W(b))=\mathbf{Q}_{\pi}$. See [13,(6.19)]. In particular, if $b^{\prime}$ is another function with the properties of $b$ then $\mathbf{Q}_{m}\left[W(b) \Delta W\left(b^{\prime}\right)\right]=0$.

As in [13, p. 53], we shall realize $X$ on the path space $W$ of the Kuznetsov process. More precisely,

$$
\Omega:=\left\{w \in W: \alpha(w)=0, Y_{\alpha+}(w) \text { exists in } E\right\} \cup\{[\Delta]\},
$$

and $X_{t}$ is the restriction of $Y_{t}$ to $\Omega$ if $t>0, X_{0}(\omega):=Y_{0+}(\omega)$ for $\omega \in \Omega$. The $\sigma$-algebras generated by $X$ are $\mathcal{F}_{t}^{\circ}:=\sigma\left\{X_{s}: 0 \leq s \leq t\right\}$ and $\mathcal{F}^{\circ}:=\sigma\left\{X_{s}: s \geq 0\right\}$. For a proof of the following result, the strong Markov property of $Y^{*}$, see [13, (6.15)]. The filtration $\left(\mathcal{G}_{t}^{m}\right)_{t \in \mathbf{R}}$ is obtained by augmenting $\left(\mathcal{G}_{t}^{0}\right)_{t \in \mathbf{R}}$ with the $\mathbf{Q}_{m}$ null sets in the usual way.

Proposition 2.1 Let $T$ be a $\left(\mathcal{G}_{t}^{m}\right)$-stopping time. Then $\mathbf{Q}_{m}$ restricted to $\mathcal{G}_{T}^{m} \cap\left\{Y_{T}^{*} \in E\right\}$ is a $\sigma$-finite measure and

$$
\mathbf{Q}_{m}\left[F \circ \theta_{T} \mid \mathcal{G}_{T}^{m}\right]=\mathbf{P}_{T}^{Y_{T}^{*}}[F], \quad \mathbf{Q}_{m} \text {-a.e. on }\left\{Y_{T}^{*} \in E\right\},
$$

for all $F \in p \mathcal{F}^{\circ}$. 
We shall also require the following form of the section theorem. Define

$$
\Lambda^{*}:=\left\{(t, w) \in \mathbf{R} \times W: Y_{t}^{*}(w) \in E\right\}
$$

evidently $\Lambda^{*}$ is $\left(\mathcal{G}_{t+}^{\circ}\right)$-optional.

Proposition 2.2 Let $\left(H_{t}\right)_{t \in \mathbf{R}}$ and $\left(K_{t}\right)_{t \in \mathbf{R}}$ be positive $\left(\mathcal{G}_{t}^{m}\right)$-optional processes. If

$$
\mathbf{Q}_{m}\left(H_{T} ; Y_{T}^{*} \in E\right)=\mathbf{Q}_{m}\left(K_{T} ; Y_{T}^{*} \in E\right)
$$

for all $\left(\mathcal{G}_{t}^{m}\right)$-stopping times $T$, then $H 1_{\Lambda^{*}}$ and $K 1_{\Lambda^{*}}$ are $\mathbf{Q}_{m}$-indistinguishable.

See [10] for a proof of Proposition 2.2.

Certain results from [9] will be crucial for our development. We recall some definitions from [9] and give precise references to the results we shall need. As

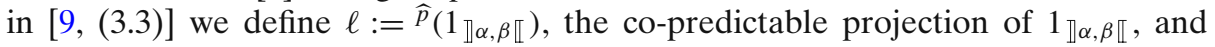
then $\Lambda:=\{\ell>0\}$. One readily checks using the argument in $[9,(3.6)]$, that $\Lambda \subset \Lambda^{*}$, modulo $\mathcal{I}^{m}$, the class of $\mathbf{Q}_{m}$-evanescent processes. See page 436 of [9]. It follows that the process $\bar{Y}$ defined in $[9,(3.8)]$ is related to $Y^{*}$ defined above in Eq. 2.3 as follows:

$$
\bar{Y}_{t}(w)= \begin{cases}Y_{t}^{*}(w), & \text { if }(t, w) \in \Lambda, \\ \Delta, & \text { if }(t, w) \notin \Lambda .\end{cases}
$$

In particular, $\Lambda=\left\{(t, w): \bar{Y}_{t}(w) \in E\right\}$. Many of the definitions and results in [9] involve $\bar{Y}$ and $\Lambda$. We shall need the extensions of these results in which $\bar{Y}$ and $\Lambda$ are replaced by $Y^{*}$ and $\Lambda^{*}$. The keys to these extensions are the strong Markov property (Proposition 2.1) and the section theorem (Proposition 2.2). Using them in place of (3.10) and (3.16)(b) in [9], the results we require are proved with only minor modifications of the arguments given in [9]. In general, we shall use the corresponding result with $Y^{*}$ and $\Lambda^{*}$ without special mention.

An arbitrary subset $A \subset E$ is m-exceptional provided $A$ is contained in a Borel $m$-polar subset $B \subset E$ with $\rho(B)=0$, where $m=\eta+\rho U$ is Riesz decomposition of $m$ into its harmonic and potential parts. It is known that $B \in \mathcal{E}$ is $m$-polar (resp. $m$-exceptional) if and only if $\left\{(t, w): Y_{t}(w) \in E\right\}\left(\operatorname{resp} .\left\{(t, w): Y_{t}^{*}(w) \in E\right\}\right)$ is $\mathbf{Q}_{m}$-evanescent. See [10]. The collection of $m$-exceptional sets is denoted by $\mathcal{N}(m)$. A property that holds off an $m$-polar set is said to hold m-quasi-everywhere or just quasi-everywhere if $m$ is understood; in symbols $m$-q.e. or just q.e.

Another key ingredient in what follows is a Ray-Knight compactification $\bar{E}$ of $E_{\Delta}$. See [19, §17-18] or [12]. Since this is a Ray-Knight compactification of $E_{\Delta}$, $\Delta$ is regarded as an ordinary trap in the definition of $\bar{E}$. Thus $U^{q}(\Delta, \cdot)=\varepsilon_{\Delta}$ and $U^{q}(x,(\{\Delta\}))=q^{-1} \mathbf{P}^{x}\left[e^{-q \zeta}\right]$ for $x \in E, q>0$. In particular $\bar{E}$ is a compact metric space in which $E_{\Delta}$ sits as a dense Borel subset, since $E$ is Lusinian. The topology $E_{\Delta}$ inherits as a subspace of $\bar{E}$ is the Ray topology of $E_{\Delta}$. Relative to this topology, $t \mapsto X_{t}$ is right continuous (rc) on [0, $\infty[$ and has left limits in $\bar{E}$ on ]0, $\infty$ [ (denoted by $\left.X_{t-}^{r}, t>0\right)$, almost surely. We emphasize that $X_{t} \in E_{\Delta}$ for $t \geq 0$ while $X_{t-}^{r} \in \bar{E}$ for $t>0$. Of course since $X$ is rc in the (original) topology of the Lusin space $E$, $t \mapsto X_{t}(\omega)$ can have at most a countable number of discontinuities in the original topology. In particular the left limit $X_{t-}$, in the original topology, exists in $E$ for all but a countable number of $t$, the exceptional $t$-set depending on $\omega$. For the 
relationship between $X_{t-}^{r}$ and $X_{t-}$ see [19, §46]. Using Proposition 2.1 one checks that $\mathbf{Q}_{m}$-a.s., $t \mapsto Y_{t}$ is Ray rc (i.e., right continuous in the Ray topology) on ] $\alpha, \infty$ [ and has Ray left limits in $\bar{E}$ on all of $\mathbf{R}$. Since $m \in$ Exc is arbitrary, we may re-define $W$ to incorporate this behavior. Therefore in the remainder of the paper we shall suppose that:

Definition 2.3 $W$ consists of all maps $w: \mathbf{R} \rightarrow E_{\Delta}$ which are $E$-valued on a nonempty open interval $] \alpha(w), \beta(w)$, take the value $\Delta$ outside this interval and are such that

(i) $w$ is rc on $] \alpha(w), \infty\left[\right.$ in the topology of $E_{\Delta}$;

(ii) $\quad w$ is Ray rc on $] \alpha(w), \infty\left[\right.$ and has left limits, $Y_{t-}^{r}(w) \in \bar{E}$ on $\mathbf{R}$.

Note that if $w \in W(b)$, then $t \mapsto Y_{t}^{*}(w)$ is right continuous on $\mathbf{R}$, in both the original topology of $E$ and in the Ray topology.

Remark 2.4 Since $X$ is Borel right process in the Ray topology, one may define $W^{r}(b)$ analogously to $W(b)$ when $E$ is given the Ray topology. In this case (iii) of (2.2) is automatically satisfied and so $W^{r}(b)$ is characterized by (i), (ii) and (iv) of (2.2) with $Y_{\alpha+}$ replaced by $Y_{\alpha+}^{r}:=\lim _{t \downarrow \alpha} Y_{t}$ where the limit is taken in the Ray topology. The decomposition $m=\eta+\pi$ into harmonic and potential parts depends only on the resolvent and so just as in the discussion below Eq. 2.3, $\mathbf{Q}_{m}\left(W(b) \Delta W^{r}(b)\right)=0$.

In general, we adhere to the usual convention that a function, $f$, defined on $E$ is extended to $\Delta$ by declaring $f(\Delta)=0$ unless explicitly stated otherwise, the construction of $\bar{E}$ being such an exception. We also write $X(t)$ for $X_{t}$ and $Y(t)$ for $Y_{t}$ when convenient. Similarly, $X(t, \omega)=X_{t}(\omega)$, etc.

\section{The Moderate Markov Dual Process}

Because of the time-symmetry of the Markov Property, the process $\left(Y_{t}, \mathbf{Q}_{m}\right)$ is a Markov process with respect to the reverse filtration $\mathcal{G}_{\geq t}^{0}:=\sigma\left\{Y_{s} ; s \geq t\right\}, t \in \mathbf{R}$. Unlike the situation in "forward" time, this process need not be a strong Markov process, but it is a moderate Markov process. In making this precise we follow [9]. However our notation differs slightly from that used there; in particular we use $Y^{*}$ in place of $\bar{Y}$, as explained in Section 2.

Let $\mathcal{G}^{m}$ be the $\mathbf{Q}_{m}$ completion of $\mathcal{G}^{\circ}$, and let $\mathcal{G}_{\geq t}^{m}$ denote the $\sigma$-algebra generated by $\mathcal{G}_{\geq t}^{\circ}$ and the $\mathbf{Q}_{m}$ null sets in $\mathcal{G}^{m}$. The co-predictable $\sigma$-algebra $\widehat{\mathcal{P}}^{\circ}$ of subsets of $\mathbf{R} \times W$ is defined by

$\widehat{\mathcal{P}}^{\circ}:=\sigma\left\{Z \in B(\mathbf{R}) \times \mathcal{G}^{\circ}: Z\right.$ is $\left(\mathcal{G}_{\geq t}^{\circ}\right)$ adapted, $t \mapsto Z_{t}$ is rc and $Z_{t}=0$ on $[\beta, \infty[\}$.

The $\mathbf{Q}_{m}$-co-predictable $\sigma$-algebra $\widehat{\mathcal{P}}^{m}$ consists of sets which differ from a copredictable set by a $\mathbf{Q}_{m}$-evanescent set. One checks that $Y^{*} \in \widehat{\mathcal{P}}^{\circ}$; see [14, p. 106]. 
A map $T: W \rightarrow[-\infty, \infty]$ is a co-stopping time (resp. $m$-co-stopping time) provided $\{T \geq t\} \in \mathcal{G}_{\geq t}^{\circ}\left(\operatorname{resp} .\{T \geq t\} \in \mathcal{G}_{\geq t}^{m}\right)$ for each $t \in \mathbf{R}$. A map $T: W \rightarrow[-\infty, \infty]$ is co-

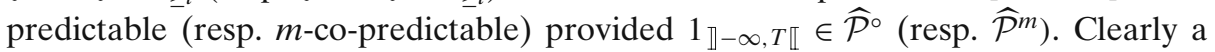
co-predictable (resp. $m$-co-predictable) $T$ is a co-stopping time (resp. $m$-co-stopping time). Associated with a co-predictable (resp. $m$-co-predictable) $T$ is the $\sigma$-algebra $\mathcal{G}_{>T}^{\circ}\left(\right.$ resp. $\left.\mathcal{G}_{>T}^{m}\right)$ generated by sets of the form $\Gamma \cap\{T<t\}$ with $\Gamma \in \mathcal{G}_{\geq t}^{0}\left(\right.$ resp. $\left.\mathcal{G}_{\geq t}^{m}\right)$, $t \in \mathbf{R}$. In the sequel we shall just make statements in the unaugmented case and leave the obvious extension to the reader.

Of course co-predictable means predictable "with time reversed". In order to make this precise define $\widehat{w}(t):=w(-t)$ for $w \in W$ and $\widehat{W}:=\{\widehat{w}: w \in W\}$. Define, for $t \in \mathbf{R}$, the coordinate maps $\widehat{Y}_{t}(\widehat{w}):=\widehat{w}(t)$ and the $\sigma$-algebras $\widehat{\mathcal{G}}^{\circ}:=\sigma\left\{\widehat{Y}_{t} ; t \in \mathbf{R}\right\}$, $\widehat{\mathcal{G}}_{t}^{\circ}:=\sigma\left\{\widehat{Y}_{s} ; s \leq t\right\}$. Define the reversal operator $r: W \rightarrow \widehat{W}$ by $r w(t):=\hat{w}(t)$. Then $r^{-1}: \widehat{W} \rightarrow W$ is given by $r^{-1} \widehat{w}(t)=\widehat{w}(-t)$. Observe that $r$ is an isomorphism between $\left(W, \mathcal{G}^{\circ}\right)$ and $\left(\widehat{W}, \widehat{\mathcal{G}}^{\circ}\right)$ with the property that $r\left(\mathcal{G}_{\geq t}^{\circ}\right)=\widehat{\mathcal{G}}_{-t}^{\circ}$ and $r^{-1} \widehat{\mathcal{G}}_{t}^{\circ}=\mathcal{G}_{\geq-t}^{\circ}$. One readily checks that $T$ is co-predictable if and only if $\widehat{T}:=-T \circ r^{-1}$ is $\left(\widehat{\mathcal{G}}_{t}^{\circ}\right)$ predictable, in which case $\mathcal{G}_{>T}^{\circ}=r^{-1} \widehat{\mathcal{G}}_{\widehat{T}-}^{\circ}$. This enables one to translate statements about predictable times into the corresponding statements about co-predictable times and conversely. For example, since $Y^{*} \in \widehat{\mathcal{P}}^{0}$, if $T$ is co-predictable and we set $Y_{T}^{*}=\Delta$ on $\{|T|=\infty\}$, then $Y_{T}^{*} \in \mathcal{G}_{>T}^{0}$. The corresponding statement in the predictable case is [4, IV-67]. (The reader is cautioned that in the 1978 English translation of [4], the statement of this result contains an unfortunate misprint.) Similarly $T$ is a co-stopping time if and only if $\widehat{T}:=-T \circ r^{-1}$ is a $\left(\widehat{\mathcal{G}}_{t}^{\circ}\right)$ stopping time. Define $\mathcal{G}_{\geq T}^{\circ}$ to consist of all $\Gamma \in \mathcal{G}^{\circ}$ such that $\Gamma \cap\{T \geq t\} \in \mathcal{G}_{\geq t}^{\circ}$ for all $t \in \mathbf{R}$. Then $\mathcal{G}_{\geq T}^{\circ}=r^{-1} \widehat{\mathcal{G}}_{\widehat{T}}^{\circ}$.

We are now prepared to describe the moderate Markov process $\widehat{X}$ associated with $X$ and $m \in$ Exc. Define

$$
\begin{gathered}
\widehat{\Omega}:=\{\beta=0\} \cup\{[\Delta]\} \subset W ; \\
\widehat{X}_{t}(\widehat{\omega}):=Y_{-t}^{*}(\widehat{\omega}), t>0, \widehat{\omega} \in \widehat{\Omega} ; \\
\widehat{\mathcal{F}}_{t}^{\circ}:=\sigma\left\{\widehat{X}_{s} ; 0<s \leq t\right\}, \widehat{\mathcal{F}}^{\circ}=\sigma\left\{\widehat{X}_{s} ; s>0\right\},
\end{gathered}
$$

and for $t \in \mathbf{R}$

$$
\check{\theta}_{t} w(s)= \begin{cases}w(t+s), & s<0, \\ \Delta, & s \geq 0\end{cases}
$$

and

$$
\widehat{\theta}_{t}=\check{\theta}_{-t} .
$$

Note that $\check{\theta}_{t}(\{t \leq \beta\}) \subset \widehat{\Omega}$ and that $\widehat{X}_{s} \circ \widehat{\theta}_{t}=\widehat{X}_{s+t}$ for $s>0, t \geq 0$. In [9], $\check{\theta}_{t}$ is denoted by $\widehat{\tau}_{t}$. It is proved in the Appendix of [9] that there exists a Borel measurable family $\left\{\widehat{\mathbf{P}}^{x}, x \in E_{\Delta}\right\}$ of probability measures on $\left(\widehat{\Omega}, \widehat{\mathcal{F}}^{\circ}\right)$ under which $\left\{\widehat{X}_{t}, t>0\right\}$ has the moderate Markov property (MMP);

$$
\widehat{\mathbf{P}}^{x}\left[F \circ \widehat{\theta}_{T} \mid \widehat{\mathcal{F}}_{T-}\right]=\widehat{\mathbf{P}}^{\widehat{X}(T)}(F) \text { on }\{\widehat{T}<\infty\},
$$


for an $\left(\widehat{\mathcal{F}}_{t}\right)$-predictable time $T$ and $F \in p \widehat{\mathcal{F}}$, where $\widehat{\mathcal{F}}$ is the intersection over all finite $\mu$ of the $\widehat{\mathbf{P}}^{\mu}$ completion of $\widehat{\mathcal{F}}^{\circ}$ and $\widehat{\mathcal{F}}_{t}$ is the usual augmentation of $\widehat{\mathcal{F}}_{t}^{\circ}$ in $\widehat{\mathcal{F}}$. $\left(\widehat{\mathbf{P}}^{\Delta}\right.$ is unit mass at $\{[\Delta]\}$.) The relationship between $\mathbf{Q}_{m}$ and $\left(\widehat{\mathbf{P}}^{x}\right)$ is expressed as follows: For each co-predictable $T, G \in p \widehat{\mathcal{G}}_{>T}^{\circ}$ and $F \in p \widehat{\mathcal{F}}^{\circ}$,

$$
\mathbf{Q}_{m}\left[F \circ \check{\theta}_{T} \cdot G ;-\infty<T<\beta\right]=\mathbf{Q}_{m}\left[\widehat{\mathbf{P}}^{Y^{*}(T)}[F] \cdot G ;-\infty<T<\beta\right] .
$$

The family $\left\{\widehat{\mathbf{P}}^{x}\right\}$ is uniquely determined modulo $m$-exceptional sets by Eqs. 3.7 and 3.8.

Note that $\widehat{\mathcal{F}}^{\circ}=\mathcal{G}^{\circ} \mid \widehat{\Omega}$ and $\widehat{\zeta}=(-\alpha) \vee 0$. Clearly $\widehat{X}$ is left continuous on $] 0, \widehat{\zeta}[$ in both the original and Ray topologies and has Ray right limits in $\bar{E}$ on [0, $\infty[$. In fact, on $\widehat{\Omega} \cap W(b), \widehat{X}$ is left continuous on ]0, $\infty$ [. However it is important to note that $\widehat{X}$ is $\left(\widehat{\mathcal{F}}_{t}^{\circ}\right)$-predictable because $Y^{*}$ is co-predictable. See the discussion following Lemma 2.9 in [13].

The moderate Markov dual semigroup $\left(\widehat{P}_{t}, t>0\right)$ and resolvent $\left(\widehat{U}^{q}, q \geq 0\right)$ are defined by

$$
\begin{aligned}
\widehat{P}_{t} f(x) & :=\widehat{\mathbf{P}}^{x}\left[f \circ \widehat{X}_{t}\right]=\widehat{\mathbf{P}}^{x}\left[f \circ \widehat{X}_{t} ; t<\widehat{\zeta}\right] \\
\widehat{U}^{q} f(x) & :=\int_{0}^{\infty} e^{-q t} \widehat{P}_{t} f(x) d t=\widehat{\mathbf{P}}^{x} \int_{0}^{\zeta} e^{-q t} f \circ \widehat{X}_{t} d t
\end{aligned}
$$

with the usual convention $f(\Delta)=0$. If $f, g \in p \mathcal{E}^{*}$ one has the duality relationships

$$
\left(P_{t} f, g\right)=\left(f, \widehat{P}_{t} g\right) \text { and }\left(U^{q} f, g\right)=\left(f, \widehat{U}^{q} g\right),
$$

where $(f, g)=\int f g d m$ whenever the integral makes sense. Of course $\widehat{P}_{t}(x, \cdot)$ and $\widehat{U}^{q}(x, \cdot)$ depend on $m$ and are uniquely determined modulo $\mathcal{N}(m)$. In what follows we shall usually omit the hat "A" where it is obviously required. For example we shall write $\widehat{\mathbf{P}}^{x}\left[f \circ X_{t}\right]$ in place of $\widehat{\mathbf{P}}^{x}\left[f \circ \widehat{X}_{t}\right]$ and $\widehat{\mathbf{P}}^{x} \int_{0}^{\infty} e^{-q t} f \circ X_{t} d t$ for $\widehat{\mathbf{P}}^{x} \int_{0}^{\infty} e^{-q t} f \circ \widehat{X}_{t} d t$.

Since $X$ is a right process $\mathbf{P}^{x}(\zeta>0)=1$ for all $x \in E$. But this may fail for the moderate Markov process $\widehat{X}$. Indeed, $\widehat{\mathbf{P}}^{x}(\zeta=0)=1$ is possible, but the set of such $x$ is $m$-polar according to the next result.

Lemma 3.1 Define $\widehat{N}:=\left\{x \in E: \widehat{\mathbf{P}}^{x}(\zeta=0)=1\right\}$. Then $\widehat{N}$ is m-polar.

Proof Observe that $\widehat{N}=\left\{x: \widehat{U}^{q} 1(x)=0\right\}$ for some, and hence every, $q \geq 0$. Let $\varphi(x):=\widehat{\mathbf{P}}^{x}(\zeta=0)$. For each $x \in E$

$$
\begin{aligned}
\widehat{U}^{q} \varphi(x) & =\widehat{\mathbf{P}}^{x} \int_{0}^{\infty} e^{-q t} \widehat{\mathbf{P}}^{X(t)}(\zeta=0) d t \\
& =\widehat{\mathbf{P}}^{x} \int_{0}^{\infty} e^{-q t} 1_{\{\zeta=t\}} d t=0 .
\end{aligned}
$$

Hence $0=q m \widehat{U}^{q} \varphi \uparrow m(\varphi)$ as $q \rightarrow \infty$. But $1_{\widehat{N}} \leq \varphi$, so $m(\widehat{N})=0$. Therefore $\widehat{\mathbf{P}}^{m}\left(X_{t} \in\right.$ $\widehat{N})=m \widehat{P}_{t}(\widehat{N}) \leq m(\widehat{N})=0$ for $t>0$. In other words $\widehat{\mathbf{P}}^{m}\left[\widehat{U}^{q} 1 \circ X_{t}=0\right]=0$ for each $t>0$. But $t \mapsto \widehat{U}^{q} 1 \circ \widehat{X}_{t}$ is left continuous on ]0, $\infty$ [, almost surely; see, for example, $[14,(2.6)]$ and the paragraph immediately following it. Consequently $\widehat{\mathbf{P}}^{m}\left(\widehat{U}^{q} 1 \circ\right.$ 
$X_{t}=0$ for some $\left.t>0\right)=0$. Combining this with Eq. 3.8 we find that $\{Y \in \widehat{N}\}$ is $\mathbf{Q}_{m}$-evanescent, and so $\widehat{N}$ is $m$-polar.

Remark It is not the case that $\widehat{N}$ is $m$-exceptional, in general, as the following example shows. Let $X$ be translation to the right at unit speed on $[0, \infty[$ and let $m=\varepsilon_{0} U$. Then $\widehat{\mathbf{P}}^{0}(\zeta=0)=1$ and $\{0\}$ is $m$-polar but not $m$-exceptional.

\section{$4 h$-Transforms}

We now fix a version of the moderate Markov kernels $\left\{\widehat{\mathbf{P}}^{x}, x \in E_{\Delta}\right\}$ and the corresponding semigroup $\left(\widehat{P}_{t}, t>0\right)$. Let $\mathcal{S}$ denote the class of excessive functions (for $X$ ) and $\mathcal{S}(m)$ the class of $h \in \mathcal{S}$ such that $h$ is Borel measurable and $h<\infty$, $m$-a.e. If $h \in \mathcal{S}(m), \xi:=h \cdot m$ is $\sigma$-finite and if $f \in p \mathcal{E}$,

$$
\xi \widehat{P}_{t} f=\left(h, \widehat{P}_{t} f\right)=\left(P_{t} h, f\right) \uparrow \xi(f) \text { as } t \downarrow 0 .
$$

Thus $\xi \in \widehat{\mathrm{Exc}}$, the class of excessive measures relative to $\left(\widehat{P}_{t}\right)$. It is known and relatively easy to check that if $\xi \in \widehat{\mathrm{Exc}}$ and $\xi \ll m$, then $\xi=h \cdot m$ with $h \in \mathcal{S}(m)$. See, for example, $[16, \S 6]$.

If $h \in \mathcal{S}(m)$, define $E_{h}:=\{0<h<\infty\} \in \mathcal{E}$. Recall that the $h$-transform semigroup $\left(P_{t}^{h}, t \geq 0\right)$ is defined on $E_{h}$ by

$$
P_{t}^{h} f(x):=h(x)^{-1} P_{t}(h f)(x), \quad x \in E_{h},
$$

and for definiteness set $P_{t}^{h}(x, \cdot)=0$ for $x \in E \backslash E_{h}$. However for our purposes the precise value on $E \backslash E_{h}$ is not particularly relevant. We refer the reader to the excellent discussion of $h$-transforms in Chapter 11 of the recent book [3]. If $h \in \mathcal{S}(m)$, $\xi=h \cdot m$ is carried by $E_{h}$ and

$$
\xi P_{t}^{h} f=m P_{t}(f h) \uparrow \xi(f) \text { as } t \downarrow 0 .
$$

Hence, using a self-explanatory notation, $\xi \in \operatorname{Exc}^{h}$. Associated with the pair $\left(\xi, P_{t}^{h}\right)$ there exists a Kuznetsov measure on $\left(W, \mathcal{G}^{\circ}\right)$ that we denote by $\mathbf{Q}_{m}^{h}$. The finitedimensional distributions of $\mathbf{Q}_{m}^{h}$ are given by

$$
\begin{aligned}
& \mathbf{Q}_{m}^{h}\left(Y_{t_{1}} \in d x_{1}, \ldots, Y_{t_{n}} \in d x_{n}\right) \\
& \quad=m\left(d x_{1}\right) P_{t_{2}-t_{1}}\left(x_{1}, d x_{2}\right) \ldots P_{t_{n}-t_{n-1}}\left(x_{n-1}, x_{n}\right) h\left(x_{n}\right),
\end{aligned}
$$

for $-\infty<t_{1}<t_{2}<\cdots<t_{n}<\infty$. This also is the Kuznetsov measure associated with the stationary entrance law $m$ and stationary exit law $h$. See [18].

The following proposition extends a familiar result on $h$-transforms to the current situation. Using the pattern of notation in Section 3, $\left(\mathcal{G}_{t}^{h \cdot m}\right)$ is the filtration obtained by augmenting $\left(\mathcal{G}_{t}^{\circ}\right)$ by all $\mathbf{Q}_{m}^{h}$ null sets in the $\mathbf{Q}_{m}^{h}$ completion $\mathcal{G}^{\circ}$. Note that $\mathcal{G}_{t}^{m} \subset \mathcal{G}_{t}^{h \cdot m}$.

Proposition 4.1 Let $T$ be a $\left(\mathcal{G}_{t}^{m}\right)$ stopping time. Then for $\Gamma \in \mathcal{G}_{T}^{m}$,

$$
\mathbf{Q}_{m}^{h}[\Gamma \cap\{-\infty<T<\beta\}]=\mathbf{Q}_{m}\left[h \circ Y_{T} ; \Gamma \cap\{-\infty<T<\beta\}\right] .
$$

Proof Decomposing $m=m_{c}+m_{d}$ into its conservative and dissipative components it suffices to prove Proposition 4.1 in the two cases separately. Also it suffices to 
suppose that $T$ is a $\left(\mathcal{G}_{t}^{\circ}\right)$ stopping time and that $\Gamma \in \mathcal{G}_{T}^{\circ}$. It is easy to see that Eq. 4.3 is valid if $T \equiv t \in \mathbf{R}$ by noting that it holds for $\Gamma=\bigcap_{j=1}^{n}\left\{Y_{t_{j}} \in B_{j}\right\}, t_{1}<\cdots<t_{n} \leq t$, $B_{j} \in \mathcal{E}$. For the general case let $a(k, n):=k 2^{-n}$ and define $T_{n}:=a(k, n)$ on $\Gamma(k, n):=$ $\{a(k-1, n) \leq T<a(k, n)\}, n \geq 1, k=0, \pm 1, \ldots$ and $T_{n}:=T$ on $\{|T|=\infty\}$. Then $T_{n} \downarrow \downarrow T$ on $\{-\infty<T<\beta\}$ and

$$
\begin{aligned}
\mathbf{Q}_{m}^{h}(\Gamma ;-\infty<T<\beta) & =\uparrow \lim _{n} \sum_{k} \mathbf{Q}_{m}^{h}(\Gamma \cap \Gamma(k, n) ; a(k, n)<\beta) \\
& =\uparrow \lim _{n} \sum_{k} \mathbf{Q}_{m}^{h}\left(\Gamma ; T_{n}=a(k, n)<\beta\right) .
\end{aligned}
$$

Since $\Gamma \in \mathcal{G}_{T}^{0},\{a(k-1, n) \leq T\} \cap(\Gamma \cap\{T<a(k, n)\}) \in \mathcal{G}_{a(k, n)}^{\circ}$ and so for each $n$ and $k$ using Eq. 4.3 for the constant time $a(n, k)$,

$$
\mathbf{Q}_{m}^{h}\left(\Gamma ; T_{n}=a(k, n)<\beta\right)=\mathbf{Q}_{m}\left(\Gamma h \circ Y_{a(k, n)} ; T_{n}=a(k, n)<\beta\right) .
$$

Therefore setting $Y_{ \pm \infty}=\Delta$ for convenience

$$
\mathbf{Q}_{m}^{h}[\Gamma ;-\infty<T<\beta]=\uparrow \lim _{n \rightarrow \infty} \mathbf{Q}_{m}\left[h \circ Y_{T_{n}} ; \Gamma\right] .
$$

Now suppose that $m$ is dissipative. Then according to the argument leading to [13, (2.14)] there exist a Borel absorbing set, $A$, carrying $m$ and an increasing sequence $\left(U f_{k}\right)$ of potentials with $f_{k} \geq 0$ and $U f_{k} \uparrow h$ on $A$. Therefore $g:=\uparrow \lim U f_{k}$ is excessive and $g=h, m$-a.e. Hence $\{g \neq h\}$ is $m$-polar and $U f_{k} \uparrow h$ except on an $m$-polar set. This in turn implies that if $T$ is a $\left(\mathcal{G}_{T}^{\circ}\right)$ stopping time, then $U f_{k} \circ Y_{T} \uparrow$ $h \circ Y_{T}, \mathbf{Q}_{m}$-a.e. Consequently,

$$
\mathbf{Q}_{m}\left[h \circ Y_{T_{n}} ; \Gamma\right]=\uparrow \lim _{k \rightarrow \infty} \mathbf{Q}_{m}\left[U f_{k} \circ Y_{T_{n}} ; \Gamma\right] .
$$

Also $\Gamma \in \mathcal{G}_{T}^{\circ} \subset \mathcal{G}_{T_{n}}^{\circ}$ since $T \leq T_{n}$ and so

$$
\mathbf{Q}_{m}\left(U f_{k} \circ Y_{T_{n}} ; \Gamma\right)=\mathbf{Q}_{m}\left(\int_{T_{n}}^{\infty} f_{k} \circ Y_{t} d t ; \Gamma\right) .
$$

Therefore each of these $\mathbf{Q}_{m}$ integrals increases with both $n$ and $k$. Hence

$$
\begin{aligned}
\mathbf{Q}_{m}^{h}(\Gamma \cap\{-\infty<T<\beta\}) & =\lim _{n} \lim _{k} \mathbf{Q}_{m}\left(\int_{T_{n}}^{\infty} f_{k} \circ Y_{t} d t ; \Gamma\right) \\
& =\lim _{k} \mathbf{Q}_{m}\left[U f_{k} \circ Y_{T} ; \Gamma\right]=\mathbf{Q}_{m}\left[h \circ Y_{T} ; \Gamma\right],
\end{aligned}
$$

establishing Eq. 4.3 for $m \in$ Dis.

It remains to prove Eq. 4.3 for $m \in$ Con. Suppose $m \in$ Con. Then $\mathbf{Q}_{m}$-a.e., $\alpha=-\infty$ and $\beta=\infty$. Also it follows from [13, (2.9)] or [13, (2.15)] that $\mathbf{Q}_{m}$-a.e., $t \mapsto$ $h \circ Y_{t}$ is constant on $\mathbf{R}$. Let $H:=\sup h \circ Y_{t}$. Then $\left\{t: h \circ Y_{t}<H\right\}$ is $\mathbf{Q}_{m}$-evanescent

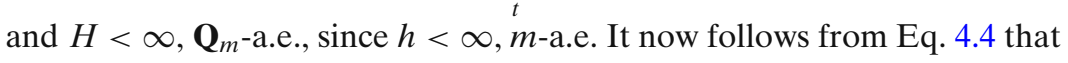

$$
\mathbf{Q}_{m}^{h}(\Gamma \cap\{-\infty<T<\beta\})=\mathbf{Q}_{m}(H ; \Gamma)=\mathbf{Q}_{m}\left(h \circ Y_{T} ; \Gamma\right),
$$

establishing Eq. 4.3 for $m \in$ Con. 
One may use the results of Section 3 for $\xi=h \cdot m, P_{t}^{h}$ and $\mathbf{Q}_{m}^{h}$ to obtain a moderate Markov family ( $\widehat{\Pi}^{x}, x \in E_{\Delta}$ ) depending on $h$ and $m$ for which the analogs of Eqs. 3.7 and 3.8 hold. But for what follows we shall need the fact that it is not necessary to introduce a new kernel; the kernel $\widehat{\mathbf{P}}^{x}$ from Section 3 suffices for all $h \in \mathcal{S}(m)$. This will be made precise in Theorem 4.4. However we shall need some preliminaries before coming to its statement. Because $\mathbf{Q}_{m}^{h}$ is $\sigma$-finite and stationary it is immediate that for each $t \in \mathbf{R}$,

$$
\mathbf{Q}_{m}^{h}(\alpha=t)=0=\mathbf{Q}_{m}^{h}(\beta=t) .
$$

In particular $\mathbf{Q}_{m}^{h}\left(Y_{t}^{*} \neq Y_{t}\right) \leq \mathbf{Q}_{m}^{h}(\alpha=t)=0$.

Lemma 4.2 If $F \in p \widehat{\mathcal{F}}^{\circ}, G \in p \mathcal{G}_{\geq t}^{0}$ and $t \in \mathbf{R}$, then

$$
\begin{aligned}
\mathbf{Q}_{m}^{h}\left[F \circ \check{\theta}_{t} \cdot G ; t<\beta\right] & =\mathbf{Q}_{m}^{h}\left[\widehat{\mathbf{P}}^{Y^{*}(t)}(F) \cdot G ; t<\beta\right] \\
& =\mathbf{Q}_{m}^{h}\left[\widehat{\mathbf{P}}^{Y(t)}(F) \cdot G ; t<\beta\right] .
\end{aligned}
$$

Proof The second equality follows immediately because $\mathbf{Q}_{m}^{h}\left(Y_{t}^{*} \neq Y_{t}\right)=0$. To see the first equality fix $t \in \mathbf{R}$. Suppose $F \in p \mathcal{F}_{t}^{\circ}, t \leq u_{1}<\cdots<u_{n}$, and $G=\prod_{i=1}^{n} g_{i} \circ Y_{u_{i}}$ where $g_{i} \in p \mathcal{E}$. Then, using Eq. 3.8 for the second equality below,

$$
\begin{aligned}
\mathbf{Q}_{m}^{h}\left[F \circ \check{\theta}_{t} \cdot G ; t<\beta\right] & =\mathbf{Q}_{m}\left[F \circ \check{\theta}_{t} \cdot G \cdot h \circ Y_{u_{n}}\right] \\
& =\mathbf{Q}_{m}\left[\widehat{\mathbf{P}}^{Y^{*}(t)}(F) \cdot G \cdot h \circ Y_{u_{n}}\right]=\mathbf{Q}_{m}^{h}\left[\widehat{\mathbf{P}}^{Y^{*}(t)}(F) \cdot G\right] .
\end{aligned}
$$

Let $0<g \leq 1, g \in \mathcal{E}$ with $m(g h)<\infty$. Fix $F \in p b \widehat{\mathcal{F}}^{\circ}$, and for $G \in p b \mathcal{G}_{\geq t}^{\circ}$ define

$$
\begin{aligned}
\mu(G) & :=\mathbf{Q}_{m}^{h}\left[F \circ \check{\theta}_{t} g \circ Y_{t} \cdot G\right] \\
\nu(G) & :=\mathbf{Q}_{m}^{h}\left[\widehat{P}^{Y^{*}(t)}(F) g \circ Y_{t} \cdot G\right] .
\end{aligned}
$$

Then $\mu$ and $v$ are finite measures on $\widehat{\mathcal{F}}^{\circ}$ which agree on $G$ of the form $G=\prod_{i=1}^{n} g_{i} \circ Y_{u_{i}}$ and hence for all $G \in b p \mathcal{G}_{\geq t}^{\circ}$. Finally let $g \uparrow 1$ through a sequence $\left(g_{k}\right)$ with $0<g_{k} \leq 1$ and $m\left(h g_{k}\right)<\infty$ for each $k$ to complete the proof of Lemma 4.2.

Before coming to the main result of this section we need to introduce a convenient metric on $\widehat{\Omega}$, following the appendix of [9]. Since $E$ is Lusin there exists a totally bounded metric $d$, say bounded by 1 , on $E$ compatible with the topology of $E$ and this is extended to $E_{\Delta}$ by setting $d(x, \Delta)=2$ for $x \in E$ and $d(\Delta, \Delta)=0$. Next, define a metric $\rho$ on $\widehat{\Omega}=\{w: \beta(w)=0\} \cup\{[\Delta]\}$ by

$$
\rho\left(w, w^{\prime}\right)=\int_{-\infty}^{0} e^{t} d=\left[w(t), w^{\prime}(t)\right] d t .
$$

Note that $\widehat{\Omega}$ consists of functions from $\mathbf{R}$ to $E_{\Delta}$ that are right continuous except at $\alpha<0$ and are constantly equal to $\Delta$ on $[0, \infty$. Thus the elements of $\widehat{\Omega}$ may be thought of as functions on $]-\infty, 0[$ and this is convenient at times. Clearly $\rho$ is a metric on $\widehat{\Omega}$ bounded by 2 and the topology induced by $\rho$ is the topology of convergence in measure relative to $\eta(d t):=e^{t} d t$ on $]-\infty, 0[$. The next lemma contains the properties of the metric space $(\widehat{\Omega}, \rho)$ that we shall need. 


\section{Lemma 4.3}

(i) $(\widehat{\Omega}, \rho)$ is separable.

(ii) If $\mathcal{B}(\widehat{\Omega})$ denotes the Borel $\sigma$-algebra associated with the metric space $(\widehat{\Omega}, \rho)$, then $\mathcal{B}(\widehat{\Omega})=\widehat{\mathcal{F}}^{0}$.

(iii) There exists a countable class $\mathcal{C}(\rho)$ of $\rho$-uniformly continuous functions from $\widehat{\Omega}$ to $[0,1]$, closed under finite products, such that $\widehat{\mathcal{F}}^{\circ}=\mathcal{B}(\widehat{\Omega})=\sigma[\mathcal{C}(\rho)]$.

Proof Property (i) is easily checked. For example let $D$ be a countable dense subset of $E_{\Delta}$ with $\Delta \in D$ and let $\mathcal{D}$ be the $D$-valued elements of $\widehat{\Omega}$ that are constant on each dyadic interval $I_{n, k}:=\left[(k-1) 2^{-n}, k 2^{-n}[, 1 \leq n \leq N,-N \leq k \leq 0, N \geq 1\right.$. Then $\mathcal{D}$ is easily seen to be dense in $\widehat{\Omega}$. For (ii) let $\overline{E_{\Delta}}$ be the compact completion of $\left(E_{\Delta}, d\right)$, and fix $f \in p C\left(\overline{E_{\Delta}}\right)$. Then $f$ is bounded and $d$-uniformly continuous on $E_{\Delta}$. For $w \in \widehat{\Omega}$, set for $n \geq 1, t \in]-\infty, 0[$,

$$
\phi_{n, t, f}(w):=n e^{-t} \int_{t}^{t+1 / n} e^{s} f \circ w(s) d s .
$$

Note that $\phi_{n, t, f}(w) \rightarrow f \circ w(t)$ as $n \rightarrow \infty$ provided $t \neq \alpha(w)$. If $w_{k} \rightarrow w$ in $\widehat{\Omega}$, then $f \circ w_{k} \rightarrow f \circ w$ in $\eta$ measure, and so $\phi_{n, t, f}\left(w_{k}\right) \rightarrow \phi_{n, t, f}(w)$ by the bounded convergence theorem. Therefore $\phi_{n, t, f}$ is continuous on $\widehat{\Omega}$, and hence $\mathcal{B}(\widehat{\Omega})$ measurable. Since $\Delta$ is isolated in $\overline{E_{\Delta}}, f:=1$ on $\overline{E_{\Delta}} \backslash\{\Delta\}$ and $f(\Delta)=0$ is uniformly continuous on $\overline{E_{\Delta}}$. Then $\phi_{t}(w):=\int_{-\infty}^{t} e^{s} f \circ w(s) d s$ is continuous on $\widehat{\Omega}$ for $t<0$. But $\{\alpha \geq t\}=$ $\left\{\phi_{t}=0\right\}$ and so $\alpha \in \mathcal{B}(\widehat{\Omega})$. Combining these observations, $1_{\{\alpha \neq t\}} f \circ Y_{t}$ is $\mathcal{B}(\widehat{\Omega})$ measurable and since $1_{\{\alpha=t\}} f \circ Y_{t}=1_{\{\alpha=t\}} f(\Delta)$, it follows that $\left.f \circ Y_{t}\right|_{\widehat{\Omega}}$ is $\mathcal{B}(\widehat{\Omega})$ measurable for $t<0$ and $f \in C\left(\overline{E_{\Delta}}\right)$. Consequently $\widehat{\mathcal{F}}^{\circ}=\left.\mathcal{G}^{\circ}\right|_{\widehat{\Omega}} \subset \mathcal{B}(\widehat{\Omega})$.

For the opposite inclusion suppose that $G \subset \widehat{\Omega}$ is open. Then there exists an increasing sequence $\left(G_{n}\right)$ of open sets with $G_{n} \subset \bar{G}_{n} \subset G_{n+1} \subset G$ with $G_{n} \uparrow G$. Let $f_{n}(w):=\left[n \rho\left(w, \bar{G}_{n}^{c}\right)\right] \wedge 1$ and note that $f_{n} \uparrow 1_{G}$. If $w^{\prime} \in G$ is fixed, $w \mapsto \rho\left(w, w^{\prime}\right)$ is $\widehat{\mathcal{F}}^{\circ}$ measurable, and since $\bar{G}_{n}^{c}$ is open, if $\mathcal{D}$ is a countable dense subset of $\widehat{\Omega}$, then

$$
w \mapsto \rho\left(w, \bar{G}_{n}^{c}\right)=\inf \left\{\rho\left(w, w^{\prime}\right): w^{\prime} \in \mathcal{D} \cap \bar{G}_{n}^{c}\right\}
$$

is $\widehat{\mathcal{F}}^{\circ}$ measurable. Therefore $G \in \widehat{\mathcal{F}}^{\circ}$ and this establishes (ii).

For (iii) let $\left(G_{n}\right)$ be a countable base for the topology of $\widehat{\Omega}$. For each $n$, there exists a sequence $\left(F_{n, k}\right)$ of $\rho$-uniformly continuous functions such that $0 \leq F_{n, k} \uparrow 1_{G_{n}}$ as $k \rightarrow \infty$. Then the closure under finite products of $\left\{F_{n, k} ; n \geq 1, k \geq 1\right\}$ has the required properties.

We now come to the main result of this section.

Theorem 4.4 Let $T$ be $\mathbf{Q}_{m}^{h}$ co-predictable, $F \in p \widehat{\mathcal{F}}^{*}, G \in \mathcal{G}_{>T}^{h \cdot m}$. Then

$$
\mathbf{Q}_{m}^{h}\left[F \circ \check{\theta}_{T} ; G \cap\{-\infty<T<\beta\}\right]=\mathbf{Q}_{m}^{h}\left[\widehat{\mathbf{P}}^{Y^{*}(T)}(F) ; G \cap\{-\infty<T<\beta\}\right] .
$$

Note that $\left\{Y_{T}^{*} \in E\right\} \subset\{-\infty<\alpha \leq T<\beta\}$.

Proof As usual it suffices to consider $T$ co-predictable, $G \in b p \mathcal{G}_{>T}^{\circ}$ and $F \in p \widehat{\mathcal{F}}^{\circ}$. There exists a sequence $\left(T_{n}\right)$ of co-predictable times each of which takes dyadic 
rational values such that $T_{n}>T$ on $\{T<\infty\}$ and $T_{n} \downarrow T, \mathbf{Q}_{m}^{h}$-a.e. See [4, IV.77] for the corresponding result for predictable times. Let $\mathbf{D}$ denote the set of dyadic rationals. For each $n$

$$
\mathbf{Q}_{m}^{h}\left[F \circ \check{\theta}_{T_{n}} \cdot G ; T_{n}<\beta\right]=\sum_{s \in \mathbf{D}} \mathbf{Q}_{m}^{h}\left[F \circ \check{\theta}_{s} \cdot G ; T_{n}=s<\beta\right] .
$$

For the moment fix $n$ and suppose that $G \in b p \mathcal{G}_{>T_{n}}^{\circ}$. If $p \geq n$, then $T_{p} \leq T_{n}$ and $G \in \mathcal{G}_{>T_{n}}^{\circ} \subset \mathcal{G}_{>T_{p}}^{\circ}$. Hence $G \cap\left\{T_{p}=s\right\} \in \mathcal{G}_{\geq s}^{\circ}$; [4, IV-56]. Bringing in Lemma 4.2 we find that for $p \geq n$

$$
\begin{aligned}
\mathbf{Q}_{m}^{h}\left[F \circ \check{\theta}_{T_{p}} \cdot G ; T_{p}<\beta\right] & =\sum_{s \in \mathbf{D}} \mathbf{Q}_{m}^{h}\left[\widehat{\mathbf{P}}^{Y^{*}(s)}(F) \cdot G ; T_{p}=s<\beta\right] \\
& =\mathbf{Q}_{m}^{h}\left[\widehat{\mathbf{P}}^{Y^{*}\left(T_{p}\right)}(F) \cdot G ; T_{p}<\beta\right] .
\end{aligned}
$$

We now adapt the argument in the appendix of [9]. Let $g \in p \mathcal{E}, g>0$ with $m(g h)<\infty$. Write Eq. 4.6 with $G$ replaced by $g \circ Y_{t} \cdot G 1_{\left\{T_{n}<t\right\}} \in p b \mathcal{G}_{>T_{n}}^{\circ}$ and $F \in$ $\mathcal{C}(\rho)$ (defined Lemma 4.3(iii)). For each $w \in W, t \mapsto \check{\theta}_{t} w$ is rc as a map from ] $-\infty, \beta(w)$ [ to $\widehat{\Omega}$. Thus letting $p \rightarrow \infty$ the left side of Eq. 4.6, with the above replacement, approaches

$$
\mathbf{Q}_{m}^{h}\left[F \circ \check{\theta}_{T} \cdot G \cdot g \circ Y_{t} 1_{\left\{T_{n}<t\right\}} ; T<\beta\right]
$$

for $F \in \mathcal{C}(\rho), \quad G \in p b \mathcal{G}_{>T_{n}}, \quad g$ as above and $t \in \mathbf{R}$. It follows from Eq. 3.8 that $t \mapsto Z_{t}:=\widehat{\mathbf{P}}^{Y^{*}(t)}(F)$ is a $\mathbf{Q}_{m}$ version of the co-predictable projection of the rc process $t \mapsto 1_{]-\infty, \beta[}(t) F \circ \breve{\theta}_{t}, \quad F \in \mathcal{C}(\rho)$. Consequently $Z$ is rc on $]-\infty, \beta\left[, \mathbf{Q}_{m}\right.$-a.e.. See Theorem 47 and the footnote on p. 120 of [5]. Moreover $Z$ is $\left(\mathcal{G}_{t}^{\circ}\right)$ adapted since $F \in \mathcal{C}(\rho)$. Now let $\left(W, \mathcal{G}_{t}^{\mathbf{Q}}, \mathcal{G}^{\mathbf{Q}}\right)$ denote the usual right continuous completion of $\left(W, \mathcal{G}_{t}^{\circ}, \mathcal{G}^{\circ}\right)$ with respect to the $\sigma$-finite measure $\mathbf{Q}:=\mathbf{Q}_{m}+\mathbf{Q}_{m}^{h}$. According to [4, IV-33], $\bar{Z}_{t}:=\lim \sup Z_{s}$ is $\left(\mathcal{G}_{t}^{\mathbf{Q}}\right)$ progressive. For $t \in \mathbf{R}$ define

$$
\Gamma:=\left\{(s, w): s \leq t<\beta(w), \bar{Z}_{s}(w) \neq Z_{s}(w)\right\},
$$

and let $\Gamma_{t}$ be the projection of $\Gamma$ on $W$. Then $\Gamma_{t} \in \mathcal{G}_{t}^{\mathbf{Q}}$ and $\mathbf{Q}_{m}\left(\Gamma_{t}\right)=0$. If $0<f \leq 1$ with $m(h f)<\infty$ then from Proposition 4.1,

$$
\mathbf{Q}_{m}^{h}\left(\Gamma_{t} \cdot f \circ Y_{t}\right)=\mathbf{Q}_{m}\left(\Gamma_{t}(f h) \circ Y_{t}\right)=0,
$$

and hence $\mathbf{Q}_{m}^{h}\left(\Gamma_{t}\right)=0$. Combining this with the same argument in which $\bar{Z}$ is replaced by $\underline{Z}_{t}:=\liminf _{s \downarrow \downarrow t} Z_{s}$, we see that $s \mapsto Z_{s}$ is rc on $]-\infty<t\left[, t<\beta, \mathbf{Q}_{m}^{h}\right.$-a.e., and since $t \in \mathbf{R}$ is arbitrary $Z$ is rc on $]-\infty, \beta$ [, $\mathbf{Q}_{m}^{h}$-a.e. Consequently the right side of Eq. 4.6 with $G$ replaced by $G \cdot g\left(Y_{t}\right) 1_{\left\{T_{n}<t\right\}}$ and $F \in \mathcal{C}(\rho)$ approaches

$$
\mathbf{Q}_{m}^{h}\left[\widehat{\mathbf{P}}^{Y^{*}(T)}(F) \cdot g \circ Y_{t} \cdot G ; T_{n}<t, T<\beta\right]
$$

as $p \rightarrow \infty$. Hence

$$
\mathbf{Q}_{m}^{h}\left[F \circ \check{\theta}_{T} g \circ Y_{t} \cdot G ; T_{n}<t, T<\beta\right]=\mathbf{Q}_{m}^{h}\left[\widehat{\mathbf{P}}^{Y^{*}(T)}[F] \cdot g \circ Y_{t} \cdot G ; T_{n}<t, T<\beta\right]
$$

for $F \in C(\rho), G \in b p \mathcal{G}_{>}, g$ as before, and $t \in \mathbf{R}$. Using the defining property of $\mathcal{C}(\rho)$, the above is then seen to hold for all $F \in p \widehat{\mathcal{F}}_{0}$. Finally let $g \uparrow 1$ through a 
sequence and then $t \uparrow \infty$ to complete the proof of Theorem 4.4, since $T_{n}<\infty$ on $\{T<\beta\}$.

\section{Ray-Knight Compactification for $\widehat{X}$}

In this section we are going to establish the existence of a Ray-Knight compactification for the dual process $\widehat{X}$. We would like to apply Theorems 8.30 and 8.45 in [3] to obtain such a compactification. However the hypotheses of those theorems are not quite satisfied. The first issue is that hypothesis (MMP) on p. 272 of [3] assumes that $t \mapsto \widehat{X}_{t}$ has right limits on [0, $\infty$ [. This is easily overcome by looking at $X$ in its Ray topology. Recall from Section 2 that $\bar{E}$ is a Ray-Knight compactification of $E_{\Delta}$ for $X$, and as pointed out in Section $3, \widehat{X}$ is left continuous on ]0, $\infty$ [ with right limits in $\bar{E}$ on $[0, \infty$ [ in the Ray topology of $X$. It is not difficult to see that this suffices for the construction. The second issue is that the key separation Theorem 8.45 requires that the resolvent be "compatible" [3, Def. 8.48], and $\left(\widehat{U}^{q}\right)$ need not be compatible. Chung and Walsh show (in [3, Lem. 8.49]) that under their hypotheses the assumption of compatibility entails no essential loss of generality. But the proof uses the fact that $\widehat{X}$ has right limits in $E$, whereas in our situation these right limits are only known to exist in $\bar{E}$. However it turns out that the proof of Theorem 8.45 goes through under our hypotheses with some minor modifications. Since this is the key technical fact for the construction and involves a beautiful argument due originally to Walsh [20], we shall give it here for the convenience of the reader. In addition the argument is somewhat simpler in the present situation. In the remainder of this section topological statements refer to the Ray topology (Definition 2.3).

Remark If it is known a priori that $\widehat{X}$ has right limits in $E$ in the original topology then one may use the original topology rather than the Ray topology in the construction of this section. For example this will be the case if $X$ has left limits in $E$ in the original topology.

Recall that $C(\bar{E})$ denotes the set of continuous functions on $\bar{E}$. The set of provisional co-branch points $\widehat{B}_{0} \subset E$ is defined as follows:

$$
E \backslash \widehat{B}_{0}:=\left\{x \in E: q \widehat{U}^{q} f(x) \rightarrow f(x) \text { as } q \rightarrow \infty \text { for all } f \in C(\bar{E})\right\} .
$$

Since $C(\bar{E})$ maybe replaced by a countable dense subset of $C(\bar{E})$ without altering $\widehat{B}_{0}$, $E \backslash \widehat{B}_{0}$ and $\widehat{B}_{0}$ are Borel subsets of $E$. Of course, in Eq. 5.1 we may replace $C(\bar{E})$ by $C_{r}(E):=\left.C(\bar{E})\right|_{E}$ since $\widehat{U}^{q}(x, \cdot)$ is a measure on $E$ for $x \in E$.

Proposition 5.1 A necessary and sufficient condition that $x \in E \backslash \widehat{B}_{0}$ is that $\widehat{\mathbf{P}}^{x}\left(X_{0+}=\right.$ $x)=1$. Of course $\widehat{X}_{0+}$ exists in $\bar{E}$.

Proof If $f \in C(\bar{E})$, then $f \circ \widehat{X}_{t} \rightarrow f \circ \widehat{X}_{0+}$ as $t \rightarrow 0$. Therefore if $x \in E$

$$
q \widehat{U}^{q} f(x)=\widehat{\mathbf{P}}^{x} \int_{0}^{\infty} e^{-t} f\left(X_{t / q}\right) d t \rightarrow \widehat{\mathbf{P}}^{x}\left[f \circ X_{0+}\right]
$$

as $q \rightarrow \infty$, which establishes Proposition 5.1 since this holds for all $f \in C(\bar{E})$. 
It is evident that the resolvent $\left(\widehat{U}^{q} ; q>0\right.$ ) separates the points of $E \backslash \widehat{B}_{0}$. Hence if $D(E)$ denotes the restrictions to $E$ of a countable dense subset of $C(\bar{E}),\left\{\widehat{U}^{1} f: f \in\right.$ $D(E)\}$ is a countable set of 1-co-potentials separating the points of $E \backslash \widehat{B}_{0}$ such that $t \mapsto \widehat{U}^{1} f \circ \widehat{X}_{t}$ is left continuous on ]0, $\infty$ [ a.s. Recall that $f \in p \mathcal{E}$ is $q$-co-supermedian provided $p \widehat{U}^{p+q} f \leq f$ for all $p>0$. We say that $f$ is co-regular provided $t \mapsto f \circ \widehat{X}_{t}$ is left continuous on ]0, $\infty$ [ a.s. A co-potential, $\widehat{U}^{q} f, q \geq 0, f \in p \mathcal{E}$ is co-regular.

Theorem 5.2 (Walsh) There exists a countable family, $\mathcal{H}$ of bounded co-regular 1-cosupermedian Borel functions on E separating the points of E.

For the proof we need a lemma whose proof we lift from [3].

Lemma 5.3 Almost surely $\left\{t: \widehat{X}_{t} \in \widehat{B}_{0}\right\}$ has Lebesgue measure zero.

Proof Fix $x \in E$. Almost surely $\widehat{\mathbf{P}}^{x}, \widehat{X}$ has at most a countable number of discontinuities, and so by the Fubini theorem, for Lebesgue a.e. $t, \widehat{X}$ is continuous at $t, \widehat{\mathbf{P}}^{x}$-a.s.. Fix such a $t>0$ and $f \in C(\bar{E})$. Then using the bounded convergence theorem for conditional expectations, $\widehat{\mathbf{P}}^{x}$-a.s.

$$
\begin{aligned}
f \circ \widehat{X}_{t} & =\lim _{n} \widehat{\mathbf{P}}^{x}\left[n \int_{0}^{\infty} e^{-n s} f \circ \widehat{X}_{t+s} d s \mid \widehat{\mathcal{F}}_{t}\right] \\
& =\lim _{n} \widehat{\mathbf{P}}^{x}\left[n \widehat{U}^{n} f \circ \widehat{X}_{t} \mid \widehat{\mathcal{F}}_{t}\right]=\lim _{n} n \widehat{U}^{n} f \circ \widehat{X}_{t} .
\end{aligned}
$$

Letting $f$ run through a countable dense subset of $C(\bar{E})$, it follows that $\widehat{X}_{t} \in E \backslash \widehat{B}_{0}$, $\widehat{\mathbf{P}}^{x}$-a.s.

Proof of Theorem 5.2 Suppose $G \subset E$ is open. Note that $\left\{\widehat{T}_{G}<t\right\}$ is the union of $\left\{\widehat{X}_{r} \in G\right\}$ over all rationals $r, 0<r<t$. Hence $\widehat{T}_{G} \in \widehat{\mathcal{F}}^{\circ}$ and so $\widehat{\mathbf{P}}\left(e^{-T_{G}}\right) \in \mathcal{E}$. (Recall the one hat convention from Section 3.) Define $\phi_{G}(x)=\widehat{\mathbf{P}}^{x}\left(e^{-T_{G}}\right)$ if $x \in E \backslash\left(\widehat{B}_{0} \cap G\right)$ and $\phi_{G}(x)=1$ if $x \in \widehat{B}_{0} \cap G$. Since $\widehat{B}_{0} \in \mathcal{E}, \phi_{G} \in \mathcal{E}$. Now Lemma 5.3 implies that $\widehat{U}^{q}\left(\cdot, \widehat{B}_{0}\right)=0$ on $E$, and this in turn implies that $q \widehat{U}^{q+1} \phi_{G} \leq \widehat{\mathbf{P}}\left(e^{-T_{G}}\right) \leq \phi_{G}$; hence $\phi_{G}$ is 1-co-supermedian. Also if $x \in G \backslash \widehat{B}_{0}$, then $\widehat{\mathbf{P}}^{x}\left[\lim _{t \downarrow 0} \widehat{X}_{t}=x\right]=1$, and so $\phi_{G}(x)=$ $\widehat{\mathbf{P}}^{x}\left(e^{-T_{G}}\right)=1$. Therefore $\phi_{G}=1$ on $G$. If $A \in \mathcal{E}$, let $\widehat{D}_{A}=\inf \left\{t \geq 0: \widehat{X}_{t} \in A\right\}$ denote the début of $A$. Note that $\widehat{D}_{G} \circ \widehat{\theta}_{t}=\widehat{T}_{G} \circ \widehat{\theta}_{t}$ on $\left\{\widehat{X}_{t} \notin G\right\}$. Therefore, if $T$ is an $\left(\widehat{\mathcal{F}}_{t}\right)$ predictable time,

$$
\widehat{\mathbf{P}}^{x}\left[\exp \left(-D_{G} \circ \theta_{T}\right) \mid \widehat{\mathcal{F}}_{T-}\right]=\phi_{G}\left(\widehat{X}_{T}\right) \text { on }\left\{\widehat{X}_{T} \notin G\right\}
$$

But if $\widehat{X}_{T} \in G, \widehat{D}_{G} \circ \widehat{\theta}_{T}=0$, so Eq. 5.2 also holds on $\left\{\widehat{X}_{T} \in G\right\}$. Now suppose $F \subset E$ is closed and choose open sets $G_{n}$ with $G_{n} \supset \bar{G}_{n+1} \supset F$ and $\cap G_{n}=F$. Define $\psi_{F}=$ $\downarrow \lim _{n} \phi_{G_{n}}$. Then $\psi_{F}$ is a Borel 1-co-supermedian function since it is the decreasing limit of a sequence of such functions. Also if $x \in \widehat{B}_{0} \cap F \subset \widehat{B}_{0} \cap G_{n}$, each $\phi_{G_{n}}(x)=1$ and so $\psi_{F}(x)=1$. On the other hand if $x \in E \backslash \widehat{B}_{0} \cap F$, then for some $n, x \in E \backslash$ 
$\widehat{B}_{0} \cap G_{n}$ and so $\psi_{F}(x) \leq \widehat{\mathbf{P}}^{x}\left[\exp \left(-T_{G_{n}}\right)\right]$. Moreover if $T$ is $\left(\widehat{\mathcal{F}}_{t}\right)$ predictable, then from Eq. 5.2

$$
\psi_{F} \circ \widehat{X}_{T}=\lim _{n} \widehat{\mathbf{P}}^{x}\left[\exp \left(-D_{G_{n}} \circ \theta_{T}\right) \mid \widehat{\mathcal{F}}_{T-}\right]=\widehat{\mathbf{P}}^{x}\left[\exp \left(-D_{F} \circ \theta_{T}\right) \mid \widehat{\mathcal{F}}_{T-}\right]
$$

since $\widehat{X}$ being left continuous implies that $\widehat{D}_{G_{n}} \uparrow \widehat{D}_{F}$.

To check that $\psi_{F}$ is co-regular, let $\left(T_{n}\right)$ be an increasing sequence of uniformly bounded $\left(\widehat{\mathcal{F}}_{t}\right)$-predictable times with limit $T$. Then using Eq. 5.3

$$
\begin{aligned}
\lim _{n} \widehat{\mathbf{P}}^{x}\left[\psi_{F} \circ X_{T_{n}}\right] & =\lim _{n} \widehat{\mathbf{P}}^{x}\left[\exp \left(-D_{F} \circ \theta_{T_{n}}\right)\right] \\
& =\widehat{\mathbf{P}}^{x}\left[\exp \left(-D_{F} \circ \theta_{T}\right)\right]=\widehat{\mathbf{P}}^{x}\left[\psi_{F} \circ X_{T}\right] .
\end{aligned}
$$

Hence by [5, VI-49], $\psi_{F} \circ \widehat{X}$ is $\widehat{\mathbf{P}}^{x}$-a.s. left continuous and so $\psi_{F}$ is co-regular.

If $x$ and $y$ are distinct points in $E$ and $\widehat{U}^{1}(x, \cdot) \neq \widehat{U}^{1}(y, \cdot)$, then the set $\left\{\widehat{U}^{1} f ; f \in\right.$ $D(E)\}$ is a countable set of 1-co-potentials separating $x$ and $y$. If $\widehat{U}^{1}(x, \cdot)=\widehat{U}^{1}(y, \cdot)$, the resolvent equation shows that $\widehat{U}^{q}(x, \cdot)=\widehat{U}^{q}(y, \cdot)$ for all $q>0$. Therefore either $x \in \widehat{B}_{0}$ or $y \in \widehat{B}_{0}$, say $x \in \widehat{B}_{0}$. Let $\mathcal{G}$ be a countable base of open sets for the (Ray) topology of $E$. By Proposition 5.1, $\widehat{\mathbf{P}}^{x}\left(X_{0+}=x\right)<1$, and so there exists $G \in \mathcal{G}$ with $x \in G, y \notin \bar{G}$ such that with positive $\widehat{\mathbf{P}}^{x}$ probability $\widehat{X}_{t} \notin G$ for $0<t<\varepsilon$ for some $\varepsilon>0$ depending on $\widehat{\omega}$; that is $\widehat{\mathbf{P}}^{x}\left(T_{G}>0\right)>0$. Then $g(x):=\widehat{\mathbf{P}}^{x}\left(e^{-T_{G}}\right)<1$. But $g$ is 1-co-excessive. Thus there exists $\left(f_{n}\right) \subset p \mathcal{E}$ with $\widehat{U}^{1} f_{n} \uparrow g$, and since $\widehat{U}^{1} f_{n}(x)=$ $\widehat{U}^{1} f_{n}(y), g(y)=g(x)<1$. Now $x \in G \cap \widehat{B}_{0}$, so $\phi_{G}(x)=1$ while $y \in E \backslash G \cap \widehat{B}_{0}$, so $\phi_{G}(y)=g(y)<1$. If $H \in \mathcal{G}$ with $x \in H \subset \bar{H} \subset G$, then $\psi_{\bar{H}}(x)=1>\psi_{\bar{H}}(y)$. Therefore

$$
\mathcal{H}:=\left\{\psi_{\bar{H}} ; H \in \mathcal{G}\right\} \cup\left\{\widehat{U}^{1} f: f \in D(E)\right\}
$$

is a family with the desired properties.

Remark The reason that we could avoid passing to a quotient space and using Lebesgue penetration times as in [3] is that there they are considering a moderate Markov process under a fixed law, $\mathbf{P}$, whereas we consider a family $\left\{\widehat{\mathbf{P}}^{x}, x \in E_{\Delta}\right\}$ and $\widehat{X}$ is moderate Markov under each $\widehat{\mathbf{P}}^{x}, x \in E_{\Delta}$.

Armed with this separation Theorem 5.2 we may now appeal to Theorem 8.30 in [3] to obtain:

Theorem 5.4 There exists a Ray-Knight compactification $\widetilde{E}$ of $E_{\Delta}$ and a Ray process $\widetilde{X}=\left(\left(\widetilde{X}_{t}\right)_{t \geq 0} ; \widetilde{\mathbf{P}}^{x}, x \in \widetilde{E}\right)$ on $\widetilde{E}$ such that for each $x \in E_{\Delta}$ the law of $\left(\widetilde{X}_{t-}\right)_{t>0}$ under $\widetilde{\mathbf{P}}^{x}$ is the same as the law of $\left(\widehat{X}_{t}\right)_{t>0}$ under $\widehat{\mathbf{P}}^{x}$.

As in the construction of $\bar{E}$ in Section 2, $\Delta$ is regarded as an ordinary trap in this construction. Therefore in applying Theorem 8.30 in [3] we need a family separating $E_{\Delta}$ and so in Theorem 5.2 we are considering $\widehat{B}_{0} \subset E_{\Delta}$. In particular $\Delta \in E_{\Delta} \backslash \widehat{B}_{0}$ since $\widehat{U}^{q} f(\Delta)=f(\Delta)$ for $q \geq 0$.

Remark Theorem 5.4 implies that in the topology of $\widetilde{E}$, the right-limit $\widehat{X}_{t+}$ exists in $\widetilde{E}$ for each $t \geq 0$, $\widehat{\mathbf{P}}^{x}$-a.s., for all $x \in E_{\Delta}$. Moreover, for each $x \in E_{\Delta}$ the $\widetilde{E}$-valued 
processes $\left(\widehat{X}_{t+}, \widehat{\mathbf{P}}^{x}\right)$ and $\left(\widetilde{X}_{t}, \widetilde{\mathbf{P}}^{x}\right)$ are equivalent. In particular, $\left(\widehat{X}_{t+}, \widehat{\mathbf{P}}^{x}\right)$ is a right continuous strong Markov process.

We refer the reader to Chapter 8 of [3] for the properties of the Ray process $\tilde{X}$ and their relationship to $\widehat{X}$. For the convenience of the reader and ease of reference we list a few of the basic properties we shall need. We employ a self-explanatory notation. Objects relative to $X$ are designated by a " " above the corresponding symbol.

$$
\begin{aligned}
& \widehat{P}_{t}(x, \cdot)=\widetilde{P}_{t}(x, \cdot) \text { and } \widehat{U}^{q}(x, \cdot)=\widetilde{U}^{q}(x, \cdot) \text { for all } x \in E_{\Delta}, t>0, \text { and } q \geq 0 . \\
& \widetilde{U}^{q}(x, \cdot) \text { is carried by } E \text { for all } x \in \widetilde{E} \backslash\{\Delta\} . \\
& \widehat{X} \text { is left continuous on }] 0, \infty[\text { with right limits in } \widetilde{E} \text { on }[0, \infty[\text { in the (Ray) } \\
& \quad \text { topology of } \widetilde{E}, \widehat{\mathbf{P}}^{x} \text {-a.s. for all } x \in E_{\Delta} \text {. } \\
& \text { If } \widetilde{C}_{b}(E) \text { denotes the bounded co-Ray continuous functions on } E \text {, then } \\
& \widehat{U}^{q} \widetilde{C}_{b}(E) \subset \widetilde{C}_{b}(E) \text { for all } q>0 \text {. }
\end{aligned}
$$

There are now three, generally distinct, topologies on $E$; the original topology and the topologies $E$ inherits from $\bar{E}$ and $\widetilde{E}$. But the three topological Borel $\sigma$-algebras on $E$ are the same and $E$ is Borel in $\bar{E}$ and in $\widetilde{E}$. We shall call the topology $E$ inherits from $\bar{E}$ (resp. $\widetilde{E}$ ) the Ray (resp. co-Ray) topology on $E$. The Ray topology on $E$ as defined in [12] maybe characterized as the weakest topology $\tau$ on $E$ satisfying $U^{q} C_{b}(E) \subset C_{b}(E, \tau)$ and $U^{q} C_{b}(E, \tau) \subset C_{b}(E, \tau)$ for each $q>0$ where $C_{b}(E)$, resp. $C_{b}(E, \tau)$, denotes the bounded continuous functions on $E$ in the original, resp. $\tau$, topology. See [12, (15.3)]. The dependence of the co-Ray topology on the choice of the separating family $\mathcal{H}$ of Theorem 5.2 seems to be an open question. We shall fix a family $\mathcal{H}$ as in Theorem 5.2 and call the resulting topology the co-Ray topology.

Define $\widehat{B} \subset E$ by

$$
E \backslash \widehat{B}:=\left\{x \in E: q \widehat{U}^{q} f(x) \rightarrow f(x) \text { as } q \rightarrow \infty \text { for all } f \in C(\widetilde{E})\right\}
$$

and call $\widehat{B}$ the set of co-branch points. Note the difference between $\widehat{B}$ and $\widehat{B}_{0}$ (defined in Eq. 5.1). Arguing exactly as for $\widehat{B}_{0}$ we obtain

Proposition $5.5 \widehat{B} \in \mathcal{E}$ and $x \in E \backslash \widehat{B}$ if and only if $\widehat{\mathbf{P}}^{x}\left(\widehat{X}_{0+}^{c r}=x\right)=1$ where $\widehat{X}_{0+}^{c r}$ denotes the right limit at 0 in $\widetilde{E}$. Almost surely $\left\{t: \widehat{X}_{t} \in \widehat{B}\right\}$ has Lebesgue measure zero.

It is easy to give an example in which $\widehat{B}_{0}$ is a proper subset of $\widehat{B}$. We do not know if it is possible for $\widehat{B}$ to be a proper subset of $\widehat{B}_{0}$.

Let $\widetilde{D}$ denote the set of non-branch points for the Ray process $\widetilde{X}$ of Theorem 5.4. It is well-known that $\widetilde{D}$ is absorbing for $\widetilde{X}$ and so one may restrict $\widetilde{X}$ to $\widetilde{D}$. Then the restricted process, $\widetilde{X} \widetilde{D}$ is a Borel right process on the Lusin space $\widetilde{D}$. Since $E \backslash \widehat{B} \subset \widetilde{D}$ and the resolvents agree on $E$ and do not charge $\widehat{B}$, one may apply the known results for the potential theory of $\widetilde{X}^{\widetilde{D}}$ to obtain results about $\widehat{X}$. For example if $\xi \in \widehat{\mathrm{Exc}}$


on $\widetilde{D}$. Here $\widetilde{\mathrm{Exc}}$ and $\widetilde{\mathrm{Har}}$ refer to the excessive and harmonic measures for the Borel right process $\widetilde{X}(\widetilde{D})$. But $\rho \leq \xi$ so $\rho$ is carried by $E \backslash \widehat{B}$, and it follows that $\rho$ is 
harmonic for $\widehat{X}$. Note, however, that we cannot assert that $\mu$ is carried by $E \backslash \widehat{B}$, and so the result is not satisfactory. This will be improved in Section 7.

\section{Entrance Laws and the Extension of Theorem 4.4}

As in Section 4 let $h \in \mathcal{S}(m)$ and $\xi:=h \cdot m$. Since $\xi \in \widehat{\operatorname{Exc}}(m)$, $\xi$ has a unique decomposition $\xi=\xi_{i}+\xi_{p}$ where $\xi \in \widehat{\operatorname{Inv}}(m)$ and $\xi_{p} \in \widehat{\operatorname{Pur}}(m)$ using the obvious notation. Since both $\xi_{p}$ and $\xi_{i}$ are absolutely continuous with respect to $m$, there exist $h_{p}$ and $h_{i}$ in $\mathcal{S}(m)$ such that $\xi_{p}=h_{p} m$ and $\xi_{i}=h_{i} m$. It is immediate that $m$-a.e., $P_{t} h_{i}=h_{i}, P_{t} h_{p} \downarrow 0$ as $t \rightarrow \infty$ and $h=h_{i}+h_{p}$. Let $\mathcal{S}_{p}(m)\left(\operatorname{resp} . \mathcal{S}_{i}(m)\right)$ denote the set of $h \in \mathcal{S}(m)$ such that $P_{t} h \downarrow 0, m$-a.e. as $t \rightarrow \infty$ (resp. $P_{t} h=h, m$-a.e. for each $\left.t\right)$. Each $h \in \mathcal{S}(m)$ may be decomposed as $h=h_{p}+h_{i}, m$-a.e., and this decomposition is unique modulo $m$ null sets. Of course two excessive functions which agree $m$-a.e. agree off an $m$-exceptional set. The elements of $\mathcal{S}_{p}(m)\left(\operatorname{resp} . \mathcal{S}_{i}(m)\right)$ are called m-purely excessive (resp. m-invariant).

Proposition 6.1 Suppose $h \in \mathcal{S}(m)$. Then $h \in \mathcal{S}_{p}(m)$ (resp. $\mathcal{S}_{i}(m)$ ) if and only if $\mathbf{Q}_{m}^{h}(\beta=\infty)=0$ (resp. $\left.\mathbf{Q}_{m}^{h}(\beta<\infty)=0\right)$. Moreover $\mathbf{Q}_{m}^{h_{p}}=\left.\mathbf{Q}_{m}^{h}\right|_{\{\beta<\infty\}}$ and $\mathbf{Q}_{m}^{h_{i}}=$ $\left.\mathbf{Q}_{m}^{h}\right|_{\{\beta=\infty\}}$. In particular $\xi_{p}(f)=\mathbf{Q}_{m}^{h}\left[f \circ Y_{0} ; \beta<\infty\right]$ and $\xi_{i}(f)=\mathbf{Q}_{m}^{h}\left[f\left(Y_{0}\right): \beta=\right.$ $\infty$ ]. Finally, $h_{p}=h \cdot \mathbf{P}^{/ h}(\zeta<\infty)$ and $h_{i}=h \cdot \mathbf{P}^{\cdot / h}(\zeta=\infty)$, m-q.e.

Proof Let $f>0$ with $m(f h)<\infty$. Then using Lemma 4.2 for the fourth equality below,

$$
\begin{aligned}
\left(P_{t} h, f\right) & =m\left(h \widehat{P}_{t} f\right)=\mathbf{Q}_{m}^{h}\left[\widehat{P}_{t} f \circ Y_{0} ; \alpha<0<\beta\right] \\
& =\mathbf{Q}_{m}^{h}\left[\widehat{\mathbf{P}}^{Y(0)}\left(f \circ \widehat{X}_{t}\right) ; \alpha<0<\beta\right]=\mathbf{Q}_{m}^{h}\left[f \circ Y_{-t} ; \alpha<0<\beta\right] \\
& =\mathbf{Q}_{m}^{h}\left[f \circ Y_{0} ; \alpha<t<\beta\right] .
\end{aligned}
$$

Letting $t \rightarrow \infty$ establishes the first assertion in Proposition 6.1. The second follows from the first because $\mathbf{Q}_{m}^{h}=\mathbf{Q}_{m}^{h_{p}}+\mathbf{Q}_{m}^{h_{i}}$. Since $\beta=\zeta \circ \theta_{0}$ on $\left\{Y_{0} \in E\right\}$, the assertions in the last sentence of Proposition 6.1 follow from those in the third sentence.

We come now to one of the main results of this section. For its statement we need a definition that is not standard but is convenient for what follows. Recall that a measure is $\Sigma$-finite provided that it is a countable sum of finite measures-called s-finite in [13].

Definition 6.2 A loose entrance law, $\widehat{v}$, for $\left(\widehat{P}_{t}\right)$ is a family $\left(\widehat{v}_{t}, t>0\right)$ of $\Sigma$-finite measures on $(E, \mathcal{E})$ such that $\widehat{v}_{t+s}=\widehat{v}_{t} \widehat{P}_{s}$ for $s, t>0$.

If each $\widehat{v}_{t}$ is $\sigma$-finite in the above definition then $\widehat{v}$ is an entrance law for $\left(\widehat{P}_{t}\right)$.

Remark If $\widehat{v}$ is a loose entrance law for $\left(\widehat{P}_{t}\right)$, then $t \mapsto \widehat{v}_{t}(f)$ is Borel measurable for $f \in p \mathcal{E}$.

We remind the reader that the Fubini theorem is valid for $\Sigma$-finite measures. 
Theorem 6.3 Let $h \in \mathcal{S}(m)$. Then

$$
\widehat{v}_{t}(f):=\mathbf{Q}_{m}^{h}\left[f \circ Y_{\beta-t}^{*} ; 0<\beta<1\right], \quad t>0, f \in p \mathcal{E},
$$

defines the unique loose entrance law for $\left(\widehat{P}_{t}\right)$ such that $\xi:=h_{p} m=\int_{0}^{\infty} \widehat{v}_{t} d t$. Moreover $\widehat{v}_{t}$ is $\sigma$-finite for Lebesgue a.e. $t$ and each $\widehat{v}_{t}$ is $\sigma$-finite on $E \backslash \widehat{N}$, where $\widehat{N}:=\{x$ : $\left.\widehat{\mathbf{P}}^{x}(\xi=0)=1\right\}$ is m-polar.

Proof Let $f \in p \mathcal{E}$. Since $\beta$ is a co-stopping time, it is $\mathcal{G}_{>\beta}^{\circ}$ measurable. See $[4,56 a]$. Now $\beta-t$ is co-predictable and $\{0<\beta<1\} \in \mathcal{G}_{>\beta}^{\circ} \subset \mathcal{G}_{>\beta-t}^{\circ}$. Therefore from Theorem 4.4,

$$
\widehat{v}_{t} \widehat{P}_{s}(f)=\mathbf{Q}_{m}^{h}\left[f \circ Y_{\beta-t-s}^{*} ; 0<\beta<1\right]=\widehat{v}_{t+s}(f) .
$$

Also,

$$
\begin{aligned}
\int_{0}^{\infty} \widehat{v}_{t}(f) d t & =\int_{0}^{\infty} \mathbf{Q}_{m}^{h}\left[f \circ Y_{\beta-t}^{*} ; 0<\beta<1\right] d t \\
& =\int_{-\infty}^{\infty} \mathbf{Q}_{m}^{h}\left[f \circ Y_{t}, 0<\beta<1\right] d t \\
& =\int_{-\infty}^{\infty} \mathbf{Q}_{m}^{h}\left[f \circ Y_{0} ; 0<\beta+t<1, \beta<\infty\right] d t=m\left(h_{p} f\right),
\end{aligned}
$$

where $f \circ Y_{t}=0$ if $t \geq \beta$ is used for the second equality and Proposition 6.1 for the last equality. Clearly each $\widehat{v}_{t}$ is $\Sigma$-finite and if $f>0$ with $m\left(h_{p} f\right)<\infty, \int_{0}^{\infty} \widehat{v}_{t}(f) d t=$ $m\left(h_{p} f\right)<\infty$ which implies that $\widehat{v}_{t}$ is $\sigma$-finite for Lebesgue a.e. $t$. Moreover

$$
\widehat{v_{t}} \widehat{U} f=\int_{t}^{\infty} \widehat{v}_{s}(f) d s \leq m\left(h_{p} f\right)<\infty .
$$

Since $\{\widehat{U} f>0\}=E \backslash \widehat{N}, \widehat{v}_{t}$ is $\sigma$-finite on $E \backslash \widehat{N}$ for each $t>0$, and by Lemma 3.1, $\widehat{N}$ is $m$-polar.

It remains to show that $\widehat{v}$ is unique. Suppose $\widehat{\mu}=\left(\widehat{\mu}_{t}, t>0\right)$ is an arbitrary loose entrance law for $\left(\widehat{P}_{t}\right)$ such that $\xi=h_{p} m=\int_{0}^{\infty} \widehat{\mu}_{t} d t$. Let $f \geq 0$ with $\xi(f)<\infty$. Then for $q>0$,

$$
\begin{aligned}
\int_{0}^{\infty} e^{-q t} \int_{t}^{\infty} \widehat{\mu}_{s}(f) d s d t & =\int_{0}^{\infty} e^{-q t} \int_{0}^{\infty} \widehat{\mu}_{s} \widehat{P}_{t} f d s d t \\
& =\int_{0}^{\infty} e^{-q t} \xi\left(\widehat{P}_{t} f\right) d t=\xi \widehat{U}^{q} f
\end{aligned}
$$

But also,

$$
\begin{aligned}
\int_{0}^{\infty} e^{-q t} \int_{t}^{\infty} \widehat{\mu}_{s}(f) d s d t & =\int_{0}^{\infty} \widehat{\mu}_{s}(f) \int_{0}^{s} e^{-q t} d t d s \\
& =q^{-1} \int_{0}^{\infty}\left(1-e^{-q s}\right) \widehat{\mu}_{s}(f) d s \\
& =q^{-1}\left[\xi(f)-\int_{0}^{\infty} e^{-q s} \widehat{\mu}_{s}(f) d s\right]
\end{aligned}
$$


Therefore

$$
\int_{0}^{\infty} e^{-q s} \widehat{\mu}_{s}(f) d s=\xi(f)-q \xi \widehat{U}^{q} f
$$

the subtractions being justified since $\xi(f)<\infty$ implies that all the terms involved are finite. Since Eq. 6.1 also holds for $\widehat{v}_{s}(f)$, we obtain $\widehat{\mu}_{t}(f)=\widehat{v}_{t}(f)$ for Lebesgue a.e. $t$ from the uniqueness theorem for Laplace transforms. But $\mathcal{E}$ is countably generated, so $\widehat{\mu}_{t}=\widehat{v}_{t}$ for a.e. $t$. If for some fixed $t>0, \widehat{\mu}_{t}=\widehat{v}_{t}$ then the entrance law property implies that $\widehat{\mu}_{s}=\widehat{v}_{s}$ for all $s \geq t$. Consequently $\widehat{\mu}_{t}=\widehat{v}_{t}$ for all $t>0$, completing the proof of Theorem 6.3.

Remark It is known under very general conditions that a purely excessive measure may be represented uniquely as the integral of an entrance law. See, for example, [17]. However with one exception all proofs known to us of both the existence and the uniqueness use the "uniqueness of charges"; that is, if $V$ is the potential kernel of the underlying semigroup and $\mu, v$ measures such that $\mu V=v V \sigma$-finite, then $\mu=v$. The one exception is the existence proof in [8]; uniqueness is not discussed there. Our existence proof is dual to that of [8]. It is easy to construct examples of processes $\widehat{X}$ of the type under consideration for which the uniqueness of charges does not hold. Nevertheless Theorem 6.3 gives the existence and the uniqueness of such a representation as the integral of a loose entrance law for $\xi \in \widehat{\operatorname{Pur}}(m)$. Note that the uniqueness argument is valid under minimal hypotheses.

The next result may be viewed as an extension of Theorem 6.3 from $E$ to $W$.

Theorem 6.4 Let $h \in \mathcal{S}(m)$. Then there exists a unique $\sigma$-finite measure $\widehat{\mathbf{Q}}_{v}$ on $\left(W, \mathcal{G}^{\circ}\right)$ carried by $\{\beta=0\}$ such that:

(i) $\mathbf{Q}_{m}^{h}\left[\Phi\left(\check{\theta}_{\beta}, \beta\right)\right]=\int \Phi(w, t) \widehat{\mathbf{Q}}_{v}(d w) d t$ for $\Phi \in p\left(\mathcal{G}^{\circ} \times \mathcal{B}(\mathbf{R})\right)$;

(ii) $\int_{-\infty}^{\infty} \sigma_{t} \widehat{\mathbf{Q}}_{v} d t=\mathbf{Q}_{m}^{h_{p}}$;

(iii) $\widehat{\mathbf{Q}}_{v}\left(\widehat{X}_{t_{1}} \in d x_{1}, \ldots, \widehat{X}_{t_{n}} \in d x_{n}\right)=\widehat{v}_{t_{1}}\left(d x_{1}\right) \widehat{P}_{t_{2}-t_{1}}\left(x_{1}, d x_{2}\right) \ldots \widehat{P}_{t_{n}-t_{n-1}}\left(x_{n-1}, d x_{n}\right)$ for $0<t_{1}<\ldots t_{n}$, where $\widehat{v}=\left(\widehat{v}_{t}, t>0\right)$ is the loose entrance law defined in Theorem 6.3.

Proof Since $\beta \circ \sigma_{t}=\beta-t, \check{\theta}_{\beta} \circ \sigma_{t}=\check{\theta}_{\beta}$. Therefore the $\sigma_{t}$ invariance of $\mathbf{Q}_{m}^{h}$ implies that

$$
\mathbf{Q}_{m}^{h}\left[\Phi\left(\check{\theta}_{\beta}, \beta\right)\right]=\mathbf{Q}_{m}^{h}\left[\Phi\left(\check{\theta}_{\beta}, \beta-t\right)\right]
$$

for $t \in \mathbf{R}$. Consequently the measure $\lambda(\Phi):=\mathbf{Q}_{m}^{h}\left[\Phi\left(\check{\theta}_{\beta}, \beta\right)\right]$ on $W \times \mathbf{R}$ is translation invariant along $\mathbf{R}$ and is clearly $\Sigma$-finite. Hence there exists a unique $\Sigma$-finite measure $\widehat{\mathbf{Q}}_{v}$ on $\left(W, \mathcal{G}^{\circ}\right)$ such that

$$
\mathbf{Q}_{m}^{h}\left[\Phi\left(\check{\theta}_{\beta}, \beta\right)\right]=\int \Phi(w, t) d t \widehat{\mathbf{Q}}_{v}(d w)
$$

for $\Phi \in p\left(\mathcal{G}^{\circ} \times \mathcal{B}(\mathbf{R})\right)$. See [13, (8.23)], for example. In particular if $F \in p\left(\mathcal{G}^{\circ}\right)$, then

$$
\widehat{\mathbf{Q}}_{\nu}(F)=\mathbf{Q}_{m}^{h}\left[F \circ \check{\theta}_{\beta}, 0<\beta<1\right] \text {. }
$$


Noting that $\beta \circ \check{\theta}_{\beta}=0$ if $\beta \in \mathbf{R}$ one sees that $\widehat{\mathbf{Q}}_{v}$ is carried by $\{\beta=0\} \subset \widehat{\Omega}$, and for $f \in p \mathcal{E}, t>0$,

$$
\widehat{\mathbf{Q}}_{v}\left[f \circ \widehat{X}_{t}\right]=\mathbf{Q}_{m}^{h}\left[f \circ Y_{\beta-t}^{*} ; 0<\beta<1\right]=\widehat{v}_{t}(f)
$$

where $\widehat{v}_{t}$ is defined in Theorem 6.3. Fix $f \in p \mathcal{E}$ with $f>0$ and $m\left(f h_{p}\right)<\infty$, define $F:=\int_{0}^{\infty} f \circ \widehat{X}_{t} d t$. Now $\widehat{\mathbf{Q}}_{v}$ is clearly $\Sigma$-finite, which justifies the interchange of integrals in computing

$$
\widehat{\mathbf{Q}}_{v}(F)=\int_{0}^{\infty} \widehat{v}_{t}(f) d t=m\left(f h_{p}\right)<\infty .
$$

Since $F>0$ on $\widehat{\Omega} \backslash\{[\Delta]\}=\{\beta=0\}, \widehat{\mathbf{Q}}_{\nu}$ is $\sigma$-finite.

For $F \in p \mathcal{G}^{\circ}$,

$$
\int_{-\infty}^{\infty} \sigma_{t} \widehat{\mathbf{Q}}_{v}(F) d t=\int_{-\infty}^{\infty} \widehat{\mathbf{Q}}_{v}\left(F \circ \sigma_{t}\right) d t=\int_{-\infty}^{\infty} \mathbf{Q}_{m}^{h}\left[F \circ \sigma_{t} \circ \check{\theta}_{\beta} ; 0<\beta<1\right] d t .
$$

Recall that $k_{t}$ denotes the killing operator defined on $W$ by $\left(k_{t} w\right)(s)=w(s)$ if $s<t$ and $\left(k_{t} w\right)(s)=\Delta$ if $s \geq t$. One verifies that $\check{\theta}_{\beta}=k_{0} \sigma_{\beta}$ and $\sigma_{t} k_{0}=k_{-t} \sigma_{t}$. Thus $\sigma_{t} \check{\theta}_{\beta}=$ $k_{-t} \sigma_{\beta+t}$. Hence, using $\beta-t=\beta \circ \sigma_{t}$ for the third equality below,

$$
\begin{aligned}
\int_{-\infty}^{\infty} \sigma_{t} \widehat{\mathbf{Q}}_{v}(F) d t & =\mathbf{Q}_{m}^{h}\left[\int_{-\infty}^{\infty} F\left[k_{-t}\left(\sigma_{\beta+t}\right)\right] d t ; 0<\beta<1\right] \\
& =\mathbf{Q}_{m}^{h}\left[\int_{-\infty}^{\infty} F\left[k_{\beta-t}\left(\sigma_{t}\right)\right] d t ; 0<\beta<1\right] \\
& =\mathbf{Q}_{m}^{h}\left[\int_{-\infty}^{\infty}\left[F \circ k_{\beta} \circ \sigma_{t} ; 0<\beta<1\right] d t\right. \\
& =\mathbf{Q}_{m}^{h}\left[\int_{-\infty}^{\infty} F \circ k_{\beta} ; 0<\beta+t<1\right] d t \\
& =\mathbf{Q}_{m}^{h}\left[F \circ k_{\beta} ; \beta \in \mathbf{R}\right]=\mathbf{Q}_{m}^{h}[F ; \beta \in \mathbf{R}],
\end{aligned}
$$

since $F \in p \mathcal{G}^{\circ}$, implying that $F \circ k_{\beta}=F$ on $\{\beta \in \mathbf{R}\}$. This establishes Theorem 6.4(ii) in view of Proposition 6.1. If $0<t_{1}<\cdots<t_{n}, f_{j} \in p \mathcal{E}, 1 \leq j \leq n$, then

$$
\begin{aligned}
\widehat{\mathbf{Q}}_{v}\left[\prod_{j=1}^{n} f_{j} \circ \widehat{X}_{t_{j}}\right] & =\mathbf{Q}_{m}^{h}\left[\prod_{j=1}^{n} f_{j} \circ Y_{\beta-t_{j}}^{*} ; 0<\beta<1\right] \\
& =\mathbf{Q}_{m}^{h}\left[f_{1} \circ Y_{\beta-t_{1}}^{*}\left(\prod_{j=2}^{n} f_{j} \circ \widehat{X}_{t_{j}-t_{1}}\right) \circ \check{\theta}_{\beta-t_{1}} ; 0<\beta<1\right] \\
& =\mathbf{Q}_{m}^{h}\left[f_{1} \circ Y_{\beta-t_{1}}^{*} \widehat{\mathbf{P}}^{Y^{*}\left(\beta-t_{1}\right)}\left(\prod_{j=2}^{n} f_{j} \circ \widehat{X}_{t_{j}-t_{1}}\right) ; 0<\beta<1\right] \\
& =\int v_{t_{1}}(d x) f_{1}(x) \widehat{\mathbf{P}}^{x}\left(\prod_{j=2}^{n} f_{j} \circ \widehat{X}_{t_{j}-t_{1}}\right)
\end{aligned}
$$


where the third equality follows from Lemma 4.3 since $\beta-t_{1}$ is co-predictable and $\beta \in \mathcal{G}_{>\beta-t_{1}}^{\circ}$. Using the fact that $\left(\widehat{X}_{t}, t>0\right)$ is Markov with transition function $\widehat{P}_{t}$ under $\widehat{\mathbf{P}}^{x}$, this establishes Theorem 6.4(iii).

Remark 6.5 It is evident that Theorem 6.4(iii) is equivalent to the statement that un$\operatorname{der} \widehat{\mathbf{Q}}_{v},\left(\widehat{X}_{t}, t>0\right)$ is Markov with semigroup $\left(\widehat{P}_{t}\right)$ and one dimensional distributions $\widehat{v}_{t}, t>0$.

We are now going to obtain a substitute for Theorem 4.4 when $T=\beta$, in analogy with Proposition 2.1. Recall the Ray-Knight compactification $\widetilde{E}$ of $E_{\Delta}$ and the discussion following Theorem 5.4. If $b \in \mathcal{E}$ with $0<b \leq 1$, define $\widehat{W}(b)$ by the following conditions:

(i) $\beta \in \mathbf{R}$ and $Y_{\beta-}^{c r} \in E$,

(ii) $\widehat{U} b \circ Y_{\beta-1 / n} \rightarrow \widehat{U} b \circ Y_{\beta-}^{c r}$ as $n \rightarrow \infty$.

We remind the reader that $Y_{\beta-}^{c r}$ denotes the left limit of $t \mapsto Y_{t}$ at $\beta$ in the co-Ray topology. Observe that $\sigma_{t}^{-1} \widehat{W}(b)=\widehat{W}(b)$ for $t \in \mathbf{R}$. Define

$$
Y_{t}^{\#}(w)= \begin{cases}Y_{\beta-}^{c r}(w) & \text { if } t=\beta(w) \text { and } w \in \widehat{W}(b), \\ Y_{t}^{*}(w) & \text { otherwise. }\end{cases}
$$

Note that $\{\beta>t\} \in \mathcal{G}_{>t}^{\circ}$ and that $\{\beta \geq t\} \in \mathcal{G}_{>t-}^{\circ}$, where $\mathcal{G}_{>t-}^{\circ}:=\cap_{s<t} \mathcal{G}_{>s}^{\circ}=\cap_{s<t} \mathcal{G}_{\geq s}^{\circ}$. Define the left germ field at $\beta$ by

$$
\mathcal{G}_{>\beta-}^{\circ}:=\left\{G \in \mathcal{G}^{\circ}: G \cap\{\beta \geq t\} \in \mathcal{G}_{>t}^{\circ} \text { for all } t \in \mathbf{R}\right\},
$$

and if $h \in \mathcal{S}(m)$ let $\mathcal{G}_{>\beta-}^{h \cdot m}$ be the usual augmentation of $\mathcal{G}_{>\beta-}^{\circ}$ by all $\mathbf{Q}_{m}^{h}$-null sets.

Theorem 6.6 Let $h \in \mathcal{S}(m)$ and fix $b$ as above such that $m\left(b h_{p}\right)<\infty$. If $G \in \mathcal{G}_{>\beta-}^{h \cdot m}$ and $F \in p \widehat{\mathcal{F}}$, then

$$
\mathbf{Q}_{m}^{h}\left[F \circ \check{\theta}_{\beta} ; G \cap \widehat{W}(b)\right]=\mathbf{Q}_{m}^{h}\left[\widehat{\mathbf{P}}^{Y^{\#}(\beta)}(F) ; G \cap \widehat{W}(b)\right] .
$$

Proof Since $\mathbf{Q}_{m}^{h_{i}}(\beta<\infty)=0$, it suffices to prove Eq. 6.5 for $h_{p}$; in the remainder of the proof we assume that $h \in \mathcal{S}_{p}(m)$. As usual it suffices the establish Eq. 6.5 for $G \in \mathcal{G}_{>\beta-}^{\circ}$ and $F \in b p \widehat{\mathcal{F}}^{\circ}$. Also observe that $\widehat{W}(b)=\left\{Y_{\beta}^{\#} \in E\right\} \in \mathcal{G}_{>\beta-}^{\circ}$. From Theorem 6.4, $\mathbf{Q}_{m}^{h}=\int_{\mathbf{R}} \sigma_{t} \widehat{\mathbf{Q}}_{v} d t$ and $\widehat{\mathbf{Q}}_{v}$ is carried by $\{\beta=0\} \subset \widehat{\Omega}$. Define $\widehat{W}_{0}(b):=$ $\widehat{W}(b) \cap\{\beta=0\}$ and $\Gamma:=G \cap\{\beta=0\}$. Then both $\widehat{W}_{0}(b)$ and $\Gamma$ are in $\left.\mathcal{G}_{>0-}^{\circ}\right|_{\widehat{\Omega}}=\widehat{\mathcal{F}}_{0+}^{\circ}$ and $\Gamma \cap \widehat{W}_{0}(b)=\Gamma \cap \widehat{W}(b)$. We shall begin by showing

$$
\widehat{\mathbf{Q}}_{v}\left[F \circ \check{\theta}_{0} ; \Gamma \cap \widehat{W}_{0}(b)\right]=\widehat{\mathbf{Q}}_{v}\left[\widehat{\mathbf{P}}^{Y^{\#}(0)}[F] ; \Gamma \cap \widehat{W}(b)\right] .
$$

Now $Y_{0}^{\#}=Y_{0-}^{c r}=\widehat{X}_{0+}^{c r} \in E$ on $\widehat{W}_{0}(b)$, and so defining $\widehat{X}_{0}:=\widehat{X}_{0+}^{c r}$ on $\widehat{W}_{0}(b)$, Eq. 6.6 becomes

$$
\widehat{\mathbf{Q}}_{v}\left[F \circ \check{\theta}_{0} ; \Gamma \cap \widehat{W}(b)\right]=\widehat{\mathbf{Q}}_{v}\left[\widehat{\mathbf{P}}^{\widehat{X}(0)}[F] ; \Gamma \cap \widehat{W}(b)\right]
$$


From the definition (6.4) of $Y^{\#}, Y_{0}^{\#}=\Delta$ on $\widehat{\Omega} \backslash \widehat{W}(b)$. Therefore, using Fatou's lemma,

$$
\begin{aligned}
\widehat{\mathbf{Q}}_{v}\left[\widehat{U} b \circ Y_{0}^{\#}\right] & =\widehat{\mathbf{Q}}_{v}\left[\widehat{U} b \circ Y_{0-}^{c r} ; \widehat{W}(b)\right] \leq \liminf _{k \rightarrow \infty} \widehat{\mathbf{Q}}_{v}\left[\widehat{U} b \circ Y_{-1 / k}\right] \\
& =\liminf _{k \rightarrow \infty} v_{1 / k} \widehat{U} b \leq m(h b)<\infty,
\end{aligned}
$$

by the choice of $b$. In particular $\widehat{U} b \circ Y_{0}^{\#}<\infty, \widehat{\mathbf{Q}}_{v}$-a.e. If $0 \leq f \in b \mathcal{E}$, define a finite measure on $\widehat{\mathcal{F}}^{\circ}=\left.\mathcal{G}_{<0}^{\circ}\right|_{\widehat{\Omega}}$ by $\mathbf{Q}^{f}(H)=\widehat{\mathbf{Q}}_{\nu}\left[H \cdot(f \widehat{U} b) \circ Y_{0}^{\#} ; \Gamma \cap \widehat{W}_{0}(b)\right], H \in \widehat{\mathcal{F}}^{\circ}$, and note that $\mathbf{Q}^{f}$ is carried by $\widehat{\Omega} \cap \widehat{W}(b)$ since $Y_{0}^{\#}=\Delta$ on $\widehat{\Omega} \backslash \widehat{W}(b)$. If $q>0$ and $g \in$ $\widetilde{C}_{b}(E)$, where $\widetilde{C}_{b}(E)$ is defined in $(5.8)$, then using Remark 6.5 and the fact that $\Gamma \in \widehat{\mathcal{F}}_{0+}^{\circ} \subset \widehat{\mathcal{F}}_{1 / k}^{\circ}$ for all $k \in \mathbf{N}$,

$$
\begin{aligned}
\int_{0}^{\infty} e^{-q t} \mathbf{Q}^{f}\left(g \circ \widehat{X}_{t}\right) d t & =\lim _{k \rightarrow \infty} \int_{1 / k}^{\infty} e^{-q t} \mathbf{Q}^{f}\left(g \circ \widehat{X}_{t-1 / k} \circ \widehat{\theta}_{1 / k}\right) d t \\
& =\lim _{k \rightarrow \infty} e^{-q / k} \mathbf{Q}^{f}\left(\widehat{U}^{q} g \circ \widehat{X}_{1 / k}\right)=\widehat{\mathbf{Q}}^{f}\left(\widehat{U}^{q} g \circ \widehat{X}_{0}\right),
\end{aligned}
$$

where the last equality comes from the bounded convergence theorem and the fact that $\widehat{\mathbf{Q}}^{f}$-a.e., $\widehat{X}_{1 / k} \rightarrow \widehat{X}_{0}$ on $\widehat{W}_{0}(b)$. Hence for $q>0$

$$
\int_{0}^{\infty} e^{-q t} \mathbf{Q}^{f}\left(g \circ \widehat{X}_{t}\right) d t=\int_{0}^{\infty} e^{-q t} \mathbf{Q}^{f}\left[\widehat{P}_{t} g \circ \widehat{X}_{0}\right] d t .
$$

Moreover $\widehat{P}_{t} g(x)=\widehat{\mathbf{P}}^{x}\left[g \circ \widehat{X}_{t}\right]$ is left continuous on $] 0, \infty[$ by (5.7). Also it follows from Eq. 6.2 that $t \mapsto g \circ \widehat{X}_{t}$ is left continuous on ]0, $\infty\left[, \widehat{\mathbf{Q}}_{v}\right.$-a.e., and so $\mathbf{Q}^{f}$-a.e. Therefore $\mathbf{Q}^{f}\left[g \circ \widehat{X}_{t}\right]=\mathbf{Q}^{f}\left[\widehat{P}_{t} g \circ \widehat{X}_{0}\right]$ for all $t>0$, first for $g \in \widetilde{C}_{b}(E)$ and then by a monotone class argument for all $g \in p \mathcal{E}$. Putting back the definition of $\mathbf{Q}^{f}$ in terms of $\widehat{\mathbf{Q}}_{\nu}$, if $g \in p \mathcal{E}$.

$$
\widehat{\mathbf{Q}}_{v}\left[g \circ \widehat{X}_{t}(f \widehat{U} b) \circ \widehat{X}_{0} ; \Gamma \cap \widehat{W}_{0}(b)\right]=\widehat{\mathbf{Q}}_{\nu}\left[\widehat{P}_{t} g \circ \widehat{X}_{0}(f \widehat{U} b) \circ \widehat{X}_{0} ; \Gamma \cap \widehat{W}_{0}(b)\right]
$$

first for $f \in p b \mathcal{E}$ and then by monotone convergence for $f \in p \mathcal{E}$. Recall $\{\widehat{U} b=0\}=$ $\widehat{N}=\{\widehat{\mathbf{P}} \cdot(\zeta=0)=1\}$. Taking $f=(\widehat{U} b)^{-1}$ we find that $\widehat{\mathbf{Q}}_{\nu}\left[g \circ \widehat{X}_{t} ; \Gamma \cap \widehat{W}_{0}(b)\right]=$ $\widehat{\mathbf{Q}}_{v}\left[\widehat{P}_{t} g \circ \widehat{X}_{0} ; \Gamma \cap \widehat{W}_{0}(b)\right]$ since on $\left\{\widehat{X}_{0} \in \widehat{N}\right\}$ both $g \circ \widehat{X}_{t}$ and $\widehat{P}_{t} g \circ \widehat{X}_{0}$ vanish for all $t>0$. Note also that $\widehat{\mathbf{Q}}_{v}$ is $\sigma$-finite on $\widehat{\mathcal{F}}_{0}^{\circ}:=\sigma\left(\widehat{X}_{0}\right)$, since $\widehat{\mathbf{Q}}_{v}\left[\widehat{U} b \circ \widehat{X}_{0}<\infty\right]$. $\widehat{\mathbf{Q}}_{v}$ is carried by $\{\beta=0\}$, so that $\widehat{\zeta}=-\alpha>0, \widehat{\mathbf{Q}}_{\nu}$-a.s. Now Eq. 6.7 and hence Eq. 6.6 follow by a standard argument using Remark 6.5.

Recalling that $h \in \mathcal{S}_{p}(m)$, and using Theorem 6.4(ii),

$$
\mathbf{Q}_{m}^{h}\left[F \circ \check{\theta}_{\beta} ; G \cap \widehat{W}(b)\right]=\int_{\mathbf{R}} \widehat{\mathbf{Q}}_{v}\left[F \circ \check{\theta}_{\beta} \circ \sigma_{t} ; \sigma_{t}^{-1}(G \cap \widehat{W}(b))\right] d t .
$$

But $\check{\theta}_{\beta} \circ \sigma_{t}=\check{\theta}_{\beta}$ and $\sigma_{t}^{-1} \widehat{W}(b)=\widehat{W}(b)$. Since $G \in \mathcal{G}_{>\beta-}^{\circ}$,

$$
\sigma_{t}^{-1} G \cap\{\beta>s\}=\sigma_{t}^{-1}(G \cap\{\beta>s-t\}) \in \sigma_{t}^{-1} \mathcal{G}_{>s-t}^{\circ}=\mathcal{G}_{>s}^{\circ}
$$

for $s \in \mathbf{R}$. Therefore $\sigma_{t}^{-1} G \in \mathcal{G}_{>\beta-}^{\circ}$. Thus, since $\beta=0, \widehat{\mathbf{Q}}_{v}$-a.e., and $Y_{\beta}^{\#} \circ \sigma_{t}=Y_{\beta}^{\#}$, the right hand side of Eq. 6.9 reduces to

$$
\int_{\mathbf{R}} \widehat{\mathbf{Q}}_{v}\left[\widehat{\mathbf{P}}^{Y^{\#}(\beta)}(F) ; \sigma_{t}^{-1}(G \cap \widehat{W}(b))\right] d t=\mathbf{Q}_{m}^{h}\left[\widehat{\mathbf{P}}^{Y^{\#}(\beta)}(F) ; G \cap \widehat{W}(b)\right],
$$


in view of Eq. 6.6. This completes the proof of Theorem 6.6.

Remark 6.7 Note that Eq. 6.7 holds for any $\Gamma \in \widehat{\mathcal{F}}_{0+}^{\circ}$. Combining this with Remark 6.5 , it follows that if $\widehat{\mathbf{Q}}_{v}$ is carried by $\widehat{W}_{0}(b)$, then $\left(\widehat{X}_{t}: t \geq 0\right)$ under $\widehat{\mathbf{Q}}_{v}$ is Markov with semigroup $\left(\widehat{P}_{t}\right)$, where of course $\widehat{X}_{0}=\widehat{X}_{0+}^{c r} \in E, \widehat{\mathbf{Q}}_{v}$-a.s.

\section{Potentials}

Recall the definition (5.9) of $\widehat{B}$, the set of co-branch points, and note that $\widehat{N} \subset \widehat{B}$, where $\widehat{N}:=\left\{x \in E: \widehat{\mathbf{P}}^{x}(\zeta=0)=1\right\}$ is the set of points in $E$ from which $\widehat{X}$ branches to $\Delta$ with probability one. Also $\widehat{N} \subset \widehat{B}_{0}$, the set of provisional co-branch points defined in Eq. 5.1. Obviously the resolvent $\left(\widehat{U}^{q}\right)$ separates the points of $E \backslash\left(\widehat{B} \cap \widehat{B}_{0}\right)$. Therefore the following uniqueness result is proved exactly as [13, Thm. 2.12].

Proposition 7.1 Let $\mu$ and $v$ be measures on $E$ which do not charge $\widehat{B} \cap \widehat{B}_{0}$, and let $q \geq 0$. If $\mu \widehat{U}^{q}$ and $\nu \widehat{U}^{q}$ are $\sigma$-finite and $\mu \widehat{U}^{q}=v \widehat{U}^{q}$, then $\mu=v$.

Before coming to the main result of this section, we need to develop some preliminary results. Recall the definition of $\widehat{W}(b)$ from Eq. 6.3 and, as in Section 6 , let $\widehat{W}_{0}(b)=\widehat{W}(b) \cap\{\beta=0\}$.

Lemma 7.2 Let $h \in \mathcal{S}_{p}(m)$ and $\xi:=h \cdot m$. Choose $0<b \leq 1$ with $m(h b)<\infty$. Then:

(i) $\mathbf{Q}_{m}^{h}$ is carried by $\widehat{W}(b)$ if and only if $\widehat{\mathbf{Q}}_{v}$ is carried by $\widehat{W}_{0}(b)$, where $\widehat{\mathbf{Q}}_{v}$ is defined in Theorem 6.4.

(ii) $\widehat{X}_{0} \in E \backslash \widehat{B}, \widehat{\mathbf{Q}}_{v}$-a.e. on $\widehat{W}_{0}(b)$ and $Y_{\beta}^{\#} \in E \backslash \widehat{B}, \mathbf{Q}_{m}^{h}$-a.e. on $\widehat{W}(b)$.

Proof The first assertion is immediate since $\mathbf{Q}_{m}^{h}=\int \sigma_{t} \widehat{\mathbf{Q}}_{v} d t$ and $\widehat{\mathbf{Q}}_{v}$ is carried by $\{\beta=0\}$. For the second let $g \in \widetilde{C}_{b}(E), 0 \leq f \leq 1$, and let $\mathbf{Q}^{f}$ be as in the proof (Theorem 6.6). Then from the definition of $\mathbf{Q}^{f}$ in terms of $\widehat{\mathbf{Q}}_{v}$ and the fact that $Y_{0}^{\#}=\widehat{X}_{0}$ on $\widehat{W}_{0}(b)$, Eq. 6.8 implies that

$$
\widehat{\mathbf{Q}}_{v}\left[\int_{0}^{\infty} e^{-q t} g \circ \widehat{X}_{t} d t(f \widehat{U} b) \circ \widehat{X}_{0} ; \widehat{W}_{0}(b)\right]=\widehat{\mathbf{Q}}_{v}\left[\widehat{U}^{q} g \circ \widehat{X}_{0}(f \widehat{U} b) \circ \widehat{X}_{0} ; \widehat{W}_{0}(b)\right] .
$$

But $\widehat{X}_{t} \rightarrow \widehat{X}_{0}=Y_{0-}^{c r}$ on $\widehat{W}_{0}(b)$ as $t \rightarrow 0$, and so multiplying by $q$ and letting $q \rightarrow \infty$, we obtain using the bounded convergence theorem

$$
\widehat{\mathbf{Q}}_{v}\left[g \circ \widehat{X}_{0}(f \widehat{U} b) \circ \widehat{X}_{0} ; \widehat{W}_{0}(b)\right]=\widehat{\mathbf{Q}}_{v}\left[\lim _{q \rightarrow \infty} q \widehat{U}^{q} g \circ \widehat{X}_{0}(f \widehat{U} b) \circ \widehat{X}_{0} ; \widehat{W}_{0}(b)\right] .
$$

Since $f \in \mathcal{E}$ with $0 \leq f \leq 1$ is arbitrary and $\widehat{W}_{0}(b) \in \widehat{\mathcal{F}}_{0+}^{\circ}, \lim _{q \rightarrow \infty} q \widehat{U}^{q} g \circ \widehat{X}_{0}=g \circ \widehat{X}_{0}$, $\widehat{\mathbf{Q}}_{v}$-a.e. on $\widehat{W}_{0}(b) \cap\left\{\widehat{U} b \circ \widehat{X}_{0}>0\right\}$. But $g \circ \widehat{X}_{0}$ and $\widehat{U} b \circ \widehat{X}_{0}$ vanish $\widehat{\mathbf{Q}}_{v}$-a.e. on $\{\widehat{U} b \circ$ $\left.\widehat{X}_{0}=0\right\}=\left\{\widehat{X}_{0} \in \widehat{N}\right\}$. This establishes the first assertion in (ii). Finally using the relation between $\mathbf{Q}_{m}^{h}$ and $\widehat{\mathbf{Q}}_{v}$ one has

$$
\mathbf{Q}_{m}^{h}\left[Y_{\beta}^{\#} \in \widehat{B} ; \widehat{W}(b)\right]=\int_{\mathbf{R}} \widehat{\mathbf{Q}}_{v}\left[\widehat{X}_{0} \in B, \widehat{W}_{0}(b)\right] d t=0,
$$


completing the proof of Lemma 7.2.

Remark Since $\widehat{\mathbf{Q}}_{v}$ is carried by $\{\beta=0\}, \widehat{\mathbf{Q}}_{\nu}\left[\widehat{W}_{0}(b) \Delta \widehat{W}(b)\right]=0$.

The next lemma is, perhaps, of some independent interest. Define $\widehat{X}_{0+}:=$ $\widehat{X}_{0+}^{c r} \in \widetilde{E}$, see Proposition 5.5 for notation. Note that on $\widehat{W}_{0}(b), \widehat{X}_{0+}=\widehat{X}_{0} \in E$.

Lemma 7.3 If $x \in E \backslash \widehat{B}$ and $f \in p \mathcal{E}$, then for $q \geq 0$

$$
\widehat{\mathbf{P}}^{x}\left[\int_{0}^{\infty} e^{-q t} f \circ X_{t} d t \mid \widehat{\mathcal{F}}_{0+}\right]=\widehat{U}^{q} f \circ \widehat{X}_{0+}, \quad \widehat{\mathbf{P}}^{x} \text {-a.s. }
$$

Proof By monotone convergence it suffices to prove this for $q>0$ and $f \in p b \mathcal{E}$. In view of Proposition 5.5, $\widehat{X}_{0+} \in E \backslash \widehat{B}, \widehat{\mathbf{P}}^{x}$-a.s. Let $\Gamma \in \widehat{\mathcal{F}}_{0+}$ and $f \in \widetilde{C}_{b}(E)$, see (5.8) for notation. Then

$$
\begin{aligned}
\widehat{\mathbf{P}}^{x}\left[\int_{0}^{\infty} e^{-q t} f \circ X_{t} d t ; \Gamma\right] & =\lim _{k \rightarrow \infty} \int_{1 / k}^{\infty} e^{-q t} \widehat{\mathbf{P}}^{x}\left[f \circ X_{t-1 / k} \circ \theta_{1 / k} ; \Gamma\right] d t \\
& =\lim _{k} e^{-q / k} \widehat{\mathbf{P}}^{x}\left[\widehat{U}^{q} f \circ X_{1 / k} ; \Gamma\right]=\widehat{\mathbf{P}}^{x}\left[\widehat{U}^{q} f \circ X_{0+} ; \Gamma\right] .
\end{aligned}
$$

Now a familiar monotone class argument shows that the extreme members of this last display are equal for $f$ in $p \mathcal{E}$ or $b \mathcal{E}$.

We come now to the main result of this section.

Theorem 7.4 Let $h \in \mathcal{S}(m)$ and suppose that $m\left(h_{p} b\right)<\infty$. Define a measure $\mu$ on E by

$$
\mu(f):=\mathbf{Q}_{m}^{h}\left[f \circ Y_{\beta}^{\#} ; 0<\beta<1, \widehat{W}(b)\right], \quad f \in p \mathcal{E} .
$$

Then $\mu(\widehat{B})=0, \mu$ is $\sigma$-finite and $\mu \widehat{U} \leq h_{p} m$. Moreover $\mu$ is the unique measure not charging $\widehat{B}$ such that $\mu \widehat{U}=h \cdot m$ if and only if $\mathbf{Q}_{m}^{h}$ is carried by $\widehat{W}(b)$.

Proof Since $\beta \in \mathbf{R}$ on $\widehat{W}(b)$, Proposition 6.1 implies that $\mu(f)=\mathbf{Q}_{m}^{h_{p}}\left[f \circ Y_{\beta}^{\#} ; 0<\right.$ $\beta<1, \widehat{W}(b)]$. Thus we shall assume that $h=h_{p}$ for the remainder of this first paragraph of the proof. By Lemma 7.2(ii), $\mu(\widehat{B})=0$. Using the relationship between $\mathbf{Q}_{m}^{h}$ and $\widehat{\mathbf{Q}}_{v}$ in Theorem 6.4(ii) and the invariance of $Y_{\beta}^{\#}$ and $\widehat{W}(b)$, one finds

$$
\begin{aligned}
\mu(f) & =\int_{\mathbf{R}} \widehat{\mathbf{Q}}_{v}\left[f \circ Y_{\beta}^{\#} ; 0<\beta-t<1, \widehat{W}(b)\right] d t \\
& =\widehat{\mathbf{Q}}_{v}\left[f \circ Y_{\beta}^{\#}, \widehat{W}(b)\right]=\widehat{\mathbf{Q}}_{v}\left[f \circ \widehat{X}_{0}, \widehat{W}(b)\right],
\end{aligned}
$$

since $\mathbf{Q}_{\nu}[\beta \neq 0]=0$, and $\widehat{X}_{0}=Y_{0}^{\#}$ on $\widehat{W}(b) \cap\{\beta=0\}$. Therefore using Remark 6.7 for the second equality

$$
\begin{aligned}
\mu \widehat{U} f & =\int_{0}^{\infty} \widehat{\mathbf{Q}}_{v}\left[\widehat{P}_{t} f \circ \widehat{X}_{0} ; \widehat{W}(b)\right] d t=\int_{0}^{\infty} \widehat{\mathbf{Q}}_{\nu}\left[f \circ \widehat{X}_{t} \circ \check{\theta}_{0} ; \widehat{W}(b)\right] d t \\
& =\int_{-\infty}^{\infty} \widehat{\mathbf{Q}}_{v}\left[f \circ Y_{t} ; \widehat{W}(b)\right] d t=\mathbf{Q}_{m}^{h}\left[f \circ Y_{0} ; \widehat{W}(b)\right] \leq \mathbf{Q}_{m}^{h}\left[f \circ Y_{0}\right]=(h \cdot m)(f),
\end{aligned}
$$


where the third equality follows because $\widehat{\mathbf{Q}}_{v}(\beta \neq 0)=0$. If $f>0$ with $m(h f)<\infty$, this shows that $\mu$ is $\sigma$-finite since $\widehat{U} f>0$ on $E \backslash \widehat{N} \supset E \backslash \widehat{B}$.

Returning to the case of general $h \in \mathcal{S}(m)$ we see that $\mu \widehat{U} \leq h_{p} m$ and that $\mu \widehat{U}=h \cdot m$ if $\mathbf{Q}_{m}^{h}$ is carried by $\widehat{W}(b)$. Since $\mu$ doesn't charge $\widehat{B}$ it is the unique such measure according to Proposition 7.1. It remains to prove the converse; that is, if $h \cdot m=\mu \widehat{U}$ for some measure $\mu$ not charging $\widehat{B}$, then $\mathbf{Q}_{m}^{h}$ is carried by $\widehat{W}(b)$. But $\mu \widehat{U}=h \cdot m$ forces $\mu$ to be $\sigma$-finite and $h \in \mathcal{S}_{p}(m)$. Define $\widehat{\mu}_{t}:=\mu \widehat{P}_{t}$ for $t>0$. Clearly $\widehat{\mu}_{t}$ is a loose entrance law for $\left(\widehat{P}_{t}\right)$, and $\int_{0}^{\infty} \widehat{\mu}_{t} d t=\mu \widehat{U}=h \cdot m$. Consequently $\widehat{\mu}_{t}=\widehat{v}_{t}$ for $t>0$ by the uniqueness assertion in Theorem 6.3. It is now evident that $\left(\widehat{X}_{t}, t>0\right)$ has the same law under $\widehat{\mathbf{Q}}_{v}$ as it has under $\widehat{\mathbf{P}}^{\mu}$. Since $\mu(\widehat{B})=0, \widehat{X}_{0+}=$ $\widehat{X}_{0+}^{c r} \in E, \widehat{\mathbf{P}}^{\mu}$-a.s. Moreover

$$
\widehat{\mathbf{P}}^{\mu} \int_{0}^{\infty} b \circ X_{s} d s=\mu \widehat{U} b=m(h b)<\infty,
$$

and this suffices to justify the use of Hunt's lemma (Th. 9.4.8 of [2]) to conclude that

$$
\widehat{U} b \circ \widehat{X}_{1 / k}=\widehat{\mathbf{P}}^{\mu}\left[\int_{1 / k}^{\infty} b \circ \widehat{X}_{s} d s \mid \widehat{\mathcal{F}}_{1 / k}\right] \rightarrow \widehat{\mathbf{P}}^{\mu}\left[\int_{0}^{\infty} b \circ \widehat{X}_{s} d s \mid \widehat{\mathcal{F}}_{0+}\right]
$$

as $k \rightarrow \infty$. Now Lemma 7.3 implies that $\widehat{U} b \circ \widehat{X}_{1 / k} \rightarrow \widehat{U} b \circ \widehat{X}_{0+}, \widehat{\mathbf{P}}^{\mu}$-a.s., and so $\widehat{\mathbf{Q}}_{v^{-}}$a.e. Consequently $\widehat{\mathbf{Q}}_{v}$ is carried by $\widehat{W}_{0}(b)$. This in turn implies that $\mathbf{Q}_{m}^{h}$ is carried by $\widehat{W}(b)$ in view of Lemma 7.2, completing the proof of Theorem 7.4.

Remark 7.5 The proof actually shows that $\left\{\widehat{X}_{t} ; t \geq 0\right\}$ has the same law under $\widehat{\mathbf{Q}}_{v}$ as it does under $\widehat{\mathbf{P}}^{\mu}$, when $\widehat{v}_{t}=\mu \widehat{P}_{t}$ for $t \geq 0$.

We are now going to describe the decomposition of $\xi \in \widehat{\operatorname{Exc}}(m)$ into potential and harmonic pieces. We need several definitions which are appropriate modifications of the corresponding concepts in the strong Markov case. See, for example, [13].

Definition 7.6 Let $\xi, \eta \in \widehat{\operatorname{Exc}}(m)$. Then $\xi$ strongly dominates $\eta$ provided $\xi=\eta+\gamma$ with $\gamma \in \widehat{\operatorname{Exc}}(m)$.

Definition 7.7 Let $\xi \in \widehat{\operatorname{Exc}}(m)$. Then $\xi$ is an $m$-co-potential (in symbols $\xi \in \widehat{\operatorname{Pot}}(m)$ ) provided $\xi=\mu \widehat{U}$ with $\mu$ not charging $\widehat{B}$, and $\xi$ is $m$-co-harmonic (in symbols $\xi \in$ $\widehat{\operatorname{Har}}(m))$ provided $\xi$ strongly dominates no non-zero $m$-co-potential.

Remark We remind the reader that the semigroup $\left(\widehat{P}_{t}\right)$ relative to which $\xi \in \widehat{\operatorname{Exc}}(m)$ is excessive depends on $m$. Also if $\xi \in \widehat{\operatorname{Pot}}(m)$, then the $\mu$ not charging $\widehat{B}$ with $\xi=\mu \widehat{U}$ is uniquely determined by Proposition 7.1, and is given by Eq. 7.1.

Theorem 7.8 Let $\xi \in \widehat{\operatorname{Exc}}(m)$; that is, $\xi=h \cdot m$ with $h \in \mathcal{S}(m)$. Let $0<b \leq 1$ with $m(h b)<\infty$. Then $\xi \in \widehat{\operatorname{Pot}}(m)$, resp. $\widehat{\operatorname{Har}}(m)$, if and only if $\mathbf{Q}_{m}^{h}$ is carried by $\widehat{W}(b)$, resp. $\widehat{W}(b)^{c}$. Finally $\xi$ has a unique decomposition $\xi=\eta+\pi$ with $\eta \in \widehat{\operatorname{Har}}(m), \pi \in$ $\widehat{\operatorname{Pot}}(m)$ and $\pi=\mu \widehat{U}$ where $\mu(f)=\mathbf{Q}_{m}^{h}\left[f \circ Y_{\beta}^{\#} ; 0<\beta<1 ; \widehat{W}(b)\right]$.

Proof Clearly Theorem 7.4 implies that $\xi \in \widehat{\operatorname{Pot}}(m)$ if and only if $\mathbf{Q}_{m}^{h}$ is carried by $\widehat{W}(b)$. Next suppose that $\mathbf{Q}_{m}^{h}(\widehat{W}(b))=0$ and $\xi=\eta+\pi$ with $\eta \in \widehat{\operatorname{Exc}}(m)$ and $\pi \in$ 
$\widehat{\operatorname{Pot}}(m)$. Then $\eta=g m$ and $\pi=p m$ with $g, p \in \mathcal{S}(m)$. Therefore $\mathbf{Q}_{m}^{h}=\mathbf{Q}_{m}^{g}+\mathbf{Q}_{m}^{p}$. Hence $\mathbf{Q}_{m}^{p}(\widehat{W}(b))=0$. But $\mathbf{Q}_{m}^{p}$ is carried by $\widehat{W}(b)$ since $\pi \in \widehat{\operatorname{Pot}}(m)$, and so $\mathbf{Q}_{m}^{p}=0$. Thus $\pi=0$; that is, $\xi \in \widehat{\operatorname{Har}}(m)$.

For the converse suppose at first that $\xi \in \widehat{\operatorname{Exc}}(m)$ is arbitrary, $\xi=h \cdot m$ with $h \in \mathcal{S}(m)$. Define measures $\pi$ and $\eta$ on $E$ by

$$
\pi(f)=\mathbf{Q}_{m}^{h}\left[f \circ Y_{0} ; \widehat{W}(b)\right] ; \quad \eta(f)=\mathbf{Q}_{m}^{h}\left[f \circ Y_{0} ; \widehat{W}(b)^{c}\right] .
$$

Clearly $\pi \leq \xi=h \cdot m$ and so $\pi \ll m$. If $t>0$, then since $\widehat{W}(b) \cap\{0<\beta\} \in \mathcal{G}_{>0}$ one has

$$
\begin{aligned}
\pi \widehat{P}_{t} f & =\mathbf{Q}_{m}^{h}\left[\widehat{P}_{t} f \circ Y_{0} ; \widehat{W}(b), 0<\beta\right]=\mathbf{Q}_{m}^{h}\left[f \circ Y_{-t} ; \widehat{W}(b), 0<\beta\right] \\
& =\mathbf{Q}_{m}^{h}\left[f \circ Y_{0} ; \widehat{W}(b), t<\beta\right] \leq \pi(f) .
\end{aligned}
$$

Therefore $\pi \in \widehat{\operatorname{Exc}}(m)$, so we have $\pi=p \cdot m$ with $p \in \mathcal{S}(m)$. Then for each $t \in \mathbf{R}$, $\mathbf{Q}_{m}^{p}\left(f \circ Y_{t}\right)=\pi(f)=\mathbf{Q}_{m}^{h}\left[f \circ Y_{t} ; \widehat{W}(b)\right]$. Now using the simple Markov property (Lemma 4.2) it follows that $\mathbf{Q}_{m}^{p}$ and $\mathbf{Q}_{m}^{h}(\cdot ; \widehat{W}(b))$ have the same finite dimensional distributions and hence $\mathbf{Q}_{m}^{p}=\mathbf{Q}_{m}^{h}(\cdot ; \widehat{W}(b))$. A similar argument shows that $\eta \in \widehat{\operatorname{Exc}}(m)$, so $\eta=g \cdot m$ with $g \in \mathcal{S}(m)$, and then $\mathbf{Q}_{m}^{g}=\mathbf{Q}_{m}^{h}\left(\cdot ; W(b)^{c}\right)$. From what has already been established we find $\pi \in \widehat{\operatorname{Pot}}(m), \eta \in \widehat{\operatorname{Har}}(m)$ and $\xi=\eta+\pi$. If $\xi \in \widehat{\operatorname{Har}}(m)$, then $\pi=0$ showing that $\mathbf{Q}_{m}^{h}(\widehat{W}(b))=0$. In the general case we have the existence of the decomposition $\xi=\pi+\eta$ and, in the light of Eq. 7.1, $\pi=\mu \widehat{U}$ with $\mu$ as claimed.

It remains to prove the uniqueness of this decomposition. Suppose $\xi=\pi^{\prime}+\eta^{\prime}$ is another such decomposition. Then using the obvious notation

$$
\mathbf{Q}_{m}^{p}=\mathbf{Q}_{m}^{h}(\cdot, \widehat{W}(b))=\mathbf{Q}_{m}^{p^{\prime}}(\cdot, \widehat{W}(b))+\mathbf{Q}_{m}^{g^{\prime}}(\cdot, \widehat{W}(b)) .
$$

But $\eta^{\prime}=g^{\prime} \cdot m \in \widehat{\operatorname{Har}}(m)$ implies that $\mathbf{Q}_{m}^{g^{\prime}}$ is carried by $\widehat{W}(b)^{c}$. Therefore $\mathbf{Q}_{m}^{p}=$ $\mathbf{Q}_{m}^{p^{\prime}}(\cdot, \widehat{W}(b))=\mathbf{Q}_{m}^{p^{\prime}}$, since $\mathbf{Q}_{m}^{p^{\prime}}$ is carried by $\widehat{W}(b)$. Hence $\pi=\pi^{\prime}$ which forces $\eta=\eta^{\prime}$.

We shall say that an excessive function $h$ is the $m$-potential of a measure $\mu$ provided $h \cdot m \in \widehat{\operatorname{Pot}}(m)$ with $h \cdot m=\mu \widehat{U}$, where $\mu(\widehat{B})=0$. We write $h=U(\mu)$ in this case and, of course, $\mu$ is unique. Similarly $h$ is $m$-harmonic provided $h \cdot m \in \widehat{\operatorname{Har}}(m)$. With these definitions we may translate Theorem 7.8 into results about excessive functions. These results should be compared with those available in the weak duality context, as found in Section 7 of [15] and in Sections 13.11 and 13.12 of [3].

Corollary 7.9 If $u \in \mathcal{S}(m)$, then $u$ may be decomposed uniquely as $u=p+h$ where $p=U(\mu)$ is an m-potential and $h$ is $m$-harmonic. Here uniqueness is up to m-polars. If $0<b \leq 1$ and $m(u b)<\infty$, and

$$
\Gamma:=\left\{0<\zeta<\infty: X_{\zeta-}^{c r} \in E, \widehat{U} b \circ X_{\zeta-1 / n} \rightarrow \widehat{U} b \circ X_{\zeta-}^{c r}\right\},
$$

then the decomposition $u=p+h$ of $u$ into m-potential and m-harmonic parts is given by $p=u \mathbf{P}^{*} / u(\Gamma)$ and $h=u \mathbf{P}^{* / u}\left(\Gamma^{c}\right)$. Moreover $u$ is an m-potential (resp. $m$-harmonic) 
if and only if $\mathbf{P}^{\bullet / u}[\Gamma]=1$ (resp. $\left.\mathbf{P}^{\bullet / u}\left[\Gamma^{c}\right]=1\right)$, m-a.e. on $\{u>0\}$. Finally, $p=U(\mu)$, where

$$
\begin{aligned}
\mu(f) & =\lim _{t \downarrow 0} t^{-1} \int_{E} u(x) \mathbf{P}^{x / u}\left[f \circ X_{\zeta-}^{c r} ; \Gamma \cap\{\zeta \leq t\}\right] m(d x) \\
& =\lim _{q \uparrow \infty} q \int_{E} u(x) \mathbf{P}^{x / u}\left[f \circ X_{\zeta-}^{c r} e^{-q \zeta} ; \Gamma\right] m(d x)
\end{aligned}
$$

Proof First note that $\Gamma \in \mathcal{F}^{\circ}$ and that $\Gamma \cap\{\zeta>t\}=\theta_{t}^{-1} \Gamma$. Therefore $x \mapsto \mathbf{P}^{x / u}(\Gamma)$ is excessive for $\left(P_{t}^{u}\right)$ and so there exists $p \in \mathcal{S}$ with $p=u \mathbf{P}^{\bullet / u}(\Gamma)$ on $\{u<\infty\}$; see $[3$, 11.17]. Since $p \leq u, m$-a.e., we have $p \in \mathcal{S}(m)$. Similarly, there exists $h \in \mathcal{S}(m)$ with $h=u \mathbf{P}^{*} / u\left[\Gamma^{c}\right]$ on $\{u<\infty\}$.

By Theorem 7.8, the potential part of $\xi:=u m$ is $\mu \widehat{U}$, where from Eq. 7.1

$$
\mu(f)=\mathbf{Q}_{m}^{u}\left(f \circ Y_{\beta-}^{c r} ;\{0<\beta<1\} \cap \widehat{W}(b)\right),
$$

since $Y_{\beta}^{\#}=Y_{\beta-}^{c r}$ on $\widehat{W}(b)$. From the second display in the proof of Theorem 7.4

$$
\mu \widehat{U}(f)=\mathbf{Q}_{m}^{u}\left(f \circ Y_{0} ; \widehat{W}(b)\right) .
$$

But for each $t \in \mathbf{R}, \widehat{W}(b)=\theta_{t}^{-1} \Gamma$ on $\{\alpha<t<\beta\}$. Thus

$$
\begin{aligned}
\mu \widehat{U}(f) & =\mathbf{Q}_{m}^{u}\left[f \circ Y_{0} ; \theta_{0}^{-1} \Gamma\right] \\
& =\mathbf{Q}_{m}^{u}\left[f \circ Y_{0} \mathbf{P}^{Y(0) / u}[\Gamma]\right]=m(p f) ;
\end{aligned}
$$

that is, $\mu \widehat{U}=p m$. It follows that $p=U(\mu)$ is the potential part of $u$. From Theorem 7.8, $u=p+g$, where $g$ is $m$-harmonic. But $u=p+h$, and since $u<\infty, m$-a.e., one has $g=h, m$-a.e., hence $m$-q.e. It remains to prove Eq. 7.3. To this end define $N(d t):=1_{\Gamma} f \circ X_{\zeta-}^{c r} \epsilon_{\zeta}(d t)$, where $f \in b p \mathcal{E}$ is fixed, and note that $N$ is an HRM on $\Omega$ as defined in Section 8 of [13]. Using the fact that $\theta_{t}^{-1} \Gamma=\widehat{W}(b)$ on $\{\alpha<t<\beta\}$, one readily checks that the extension $N^{*}$ of $N$ to $W$ defined in $[13,(8.18)]$ is given by $N^{*}(d t)=1_{\widehat{W}(b)} f \circ Y_{\beta-}^{c r} \epsilon_{\beta}(d t)$. Let $v_{N}^{\xi}$ denote the characteristic measure of $N$ relative to the $u$-transform of $X$ and $\xi=u m \in \operatorname{Exc}^{u}$. Using Theorem (8.21) of [13] with $\varphi=1_{] 0,1[}$, gives

$$
v_{N}^{\xi}(1)=\mathbf{Q}_{m}^{u}\left[f \circ Y_{\beta-}^{c r} ; \widehat{W}(b) \cap\{0<\beta<1\}\right]=\mu(f) .
$$

But from $[13,(8.9)]$ one also has

$$
\begin{aligned}
v_{N}^{\xi}(1) & =\lim _{t \downarrow 0} t^{-1} \int_{E} u(x) \mathbf{P}^{x / u}\left[f \circ X_{\zeta-}^{c r} ; \Gamma \cap\{\zeta \leq t\}\right] m(d x) \\
& =\lim _{q \uparrow \infty} q \int_{E} u(x) \mathbf{P}^{x / u}\left[f \circ X_{\zeta-}^{c r} e^{-q \zeta} ; \Gamma\right] m(d x),
\end{aligned}
$$

which establishes Eq. 7.3.

\section{Co-balayage and Walsh's Interior Réduite}

In [8] the authors introduced a balayage operation associated with a stationary stopping time. This is also discussed in Section 7 of [13]. We are going to define a co- 
balayage operation associated with a class of stationary co-stopping times. Applying this to a specific stationary co-stopping time will lead to a generalization of the interior réduite discussed in Section 13.12 of [3].

Let $\mathbf{Q}$ be a finite (or $\sigma$ - finite) measure on $\left(W, \mathcal{G}^{\circ}\right)$ and let $\mathcal{G}_{>t}^{\mathbf{Q}}$ be the "co-filtration" obtained by augmenting $\left(\mathcal{G}_{\geq t}^{\circ}\right)$ by all $\mathbf{Q}$ null sets in $\mathcal{G}^{\mathbf{Q}}$, the $\mathbf{Q}$-completion of $\mathcal{G}^{\circ}$. Define $\mathcal{G}_{\geq t}^{*}:=\cap_{\mathbf{Q}} \mathcal{G}_{\geq t}^{\mathbf{Q}}$ where the intersection is over all finite measures on $\mathbf{Q}$ on $\mathcal{G}^{\circ} . \mathcal{G}_{>t}^{*}, \mathcal{G}_{t}^{*}$, etc. are defined similarly.

Definition 8.1 A universal co-stopping time $S$ is a map $S: W \rightarrow[-\infty, \infty]$ such that $\{S>t\} \in \widehat{\mathcal{G}}_{>t}^{*}$ for each $t \in \mathbf{R}$. If, in addition, $t+S \circ \sigma_{t}=S$, then $S$ is a stationary universal co-stopping time. The class of such times is denoted by $\mathbf{S}\left(\mathcal{G}_{>t}^{*}\right)$.

Remark Since $\{S>t\}=\cup_{n}\left\{S \geq t+\frac{1}{n}\right\}$ one may replace $\mathcal{G}_{>t}^{*}$ by $\mathcal{G}_{\geq t}^{*}$ and obtain the same class of universal co-stopping times. Recall the definition of killing operators on $W, k_{t} w(s)=w(s)$ if $s<t$ and $k_{t} w(s)=\Delta$ if $s \geq t$.

We now define the co-balayage, $\widehat{R}_{S} \xi$, of $\xi=h \cdot m \in \widehat{\operatorname{Exc}}(m)$ on $S$ a stationary universal co-stopping time,

$$
\widehat{R}_{S} \xi(f)=\widehat{R}_{S}(h \cdot m)(f):=\mathbf{Q}_{m}^{h}\left[f \circ Y_{t} ; S>t\right]=\mathbf{Q}_{m}^{h}\left[f \circ Y_{t} \circ k_{S}\right]
$$

Clearly $\widehat{R}_{S} \xi$ does not depend on the choice of $t$ in Eq. 8.1 and $\widehat{R}_{S} \xi$ is a measure with $\widehat{R}_{S} \xi \leq \xi$, in particular $\widehat{R}_{S} \xi \ll m$. Also

$$
\begin{aligned}
\widehat{R}_{S} \xi\left(\widehat{P}_{t} f\right) & =\mathbf{Q}_{m}^{h}\left[\widehat{P}_{t} f \circ Y_{0} ; S>0\right]=\mathbf{Q}_{m}^{h}\left[f \circ Y_{-t} ; S>0\right] \\
& =\mathbf{Q}_{m}^{h}\left[f \circ Y_{0} ; S>t\right] \uparrow \widehat{R}_{S} \xi(f)
\end{aligned}
$$

as $t \downarrow 0$. Therefore $\widehat{R}_{S} \xi \in \widehat{\operatorname{Exc}}(m)$ and so $\widehat{R}_{S}(h \cdot m)=p_{S} h \cdot m$ with $p_{S} h \in \mathcal{S}(m)$ where $p_{S} h \leq h$ q.e.. Of course, $p_{S} h$ is only determined q.e. Moreover, $p_{S}(g+h)=$ $p_{S} g+p_{S} h$ q.e. if $g, h \in \mathcal{S}(m)$.

Proposition 8.2 With the above notation $\mathbf{Q}_{m}^{p_{S} h}(F)=\mathbf{Q}_{m}^{h}\left[F \circ k_{S}: S>\alpha\right]$ for $F \in p \mathcal{G}_{0}^{\circ}$. 
Proof Suppose $F=\prod_{j=1}^{n} f_{j} \circ Y_{t_{j}}$, where $t_{1}<\cdots<t_{n}$ and $f_{j} \in p \mathcal{E}$ for $j \geq 1$. Then $F \circ$ $k_{S}=F$ or 0 according as $t_{n}<S$ or $t_{n} \geq S$. Writing $G:=\prod_{j=1}^{n-1} f_{j} \circ \widehat{X}_{t_{n}-t_{j}}$, we compute

$$
\begin{aligned}
\mathbf{Q}_{m}^{h}\left[F \circ k_{S} ; S>\alpha\right] & =\mathbf{Q}_{m}^{h}\left[\prod_{j=1}^{n} f_{j} \circ Y_{t_{j}} ; t_{n}<S\right] \\
& =\mathbf{Q}_{m}^{h}\left[f_{n} \circ Y_{t_{n}} \widehat{\mathbf{P}}^{Y\left(t_{n}\right)}(G) ; t_{n}<S\right] \\
& =\int \widehat{R}_{S}(h \cdot m)(d x) f_{n}(x) \widehat{\mathbf{P}}^{x}(G) \\
& =\left(f_{n} p_{S} h, \widehat{\mathbf{P}}^{\bullet}(G)\right) \\
& =\left(f_{n} p_{S} h, \widehat{P}_{t_{n}-t_{n-1}} f_{n-1} \cdots \widehat{P}_{t_{2}-t_{1}} f_{1}\right) \\
& =\left(f_{1}, P_{t_{2}-t_{1}} f_{2} \cdots P_{t_{n}-t_{n-1}}\left(f_{n} p_{S} h\right)\right) \\
& =\mathbf{Q}_{m}^{p_{S} h}(F) .
\end{aligned}
$$

Thus $\mathbf{Q}_{m}^{p_{s} h}$ and $k_{S} \mathbf{Q}_{m}^{h}[\cdot ; S>\alpha]$ have the same finite dimensional distributions and both vanish on $\{[\Delta]\}$. Hence they agree since a Kuznetsov measure is uniquely determined by its finite dimensional distributions and the fact that it does not charge $\{[\Delta]\}$.

Remark If $F([\Delta])=0$, then $\mathbf{Q}_{n}^{p_{S} h}[F]=\mathbf{Q}_{m}^{h}\left[F \circ k_{S}\right]$.

Proposition 8.2 and the next result are analogous to (7.5) in [13].

Proposition 8.3 Let $S \in \mathbf{S}\left(\mathcal{G}_{>t}^{*}\right)$ and suppose that $S \leq \beta$. If $\xi=h \cdot m \in \widehat{\operatorname{Exc}}(m)$, then

(i) $\left(\widehat{R}_{S} \xi\right)_{p}(f)=\mathbf{Q}_{m}^{h}\left[f \circ Y_{0} ; 0<S<\infty\right]=\int_{0}^{\infty} \widehat{v}_{t}^{S}(f) d t$, where $\widehat{v}_{t}^{S}(f)=\mathbf{Q}_{m}^{h}\left[f \circ Y_{S-t}^{*}\right.$; $0<S<1]$.

(ii) The potential part of $\widehat{R}_{S} \xi$ equals $\mu_{S} \widehat{U}$ where $\mu_{S}(f)=\mathbf{Q}_{m}^{h}\left[f \circ Y_{S-}^{c r} ; 0<S<1\right.$, $\left.k_{S}^{-1} \widehat{W}(b)\right]$.

Proof Since $\beta \circ k_{S}=\beta \wedge S=S$ under the hypothesis, (i) follows from Proposition 6.1, Theorem 6.3 and Proposition 8.2 and (ii) from Theorem 7.8 and Proposition 8.2.

We are now going to consider a specific example. Let $A \in \mathcal{E}$ and define ( $\sup \phi=$ $-\infty$ by convention)

$$
S_{A}:=\sup \left\{t: Y_{t-}^{c r} \in A\right\}=\sup \left\{t>\alpha: Y_{t-}^{c r} \in A\right\} .
$$

Note that $S_{A} \leq \beta, S_{A}>\alpha$ on $\left\{S_{A}>-\infty\right\}$, and that $Y_{t-}^{c r}=Y_{t-}^{* c r}=\Delta$ if $t \leq \alpha$. Clearly $t+S_{A} \circ \sigma_{t}=S_{A}$. Since $A \in \mathcal{E}, A$ is Borel in $\widetilde{E}$. See Theorem 5.4 for notation. Therefore

$$
\left\{(s, w): s>t, Y_{s-}^{c r}(w) \in A\right\} \in \mathcal{B}_{>t} \otimes \mathcal{G}_{>t}^{\circ}
$$


where $\mathcal{B}_{>t}$ denotes the Borel $\sigma$-algebra of $] t, \infty\left[\right.$. But $\left\{S_{A}>t\right\}$ is the projection of this set on $W$ and so $\left\{S_{A}>t\right\} \in \mathcal{G}_{>t}^{*}$. See III-82b of [4]. Therefore $S_{A} \in \mathbf{S}\left(\mathcal{G}_{>t}^{*}\right)$. If $\xi=h \cdot m \in \widehat{\operatorname{Exc}}(m)$ we write $\widehat{R}_{A} \xi=\widehat{R}_{S_{A}} \xi$ and $p_{A} h=p_{S_{A}} h$.

Proposition 8.4 Let $T_{A}^{-}:=\inf \left\{t>0: X_{t-}^{c r} \in A\right\}$. Then using the above notation $p_{A} h=h \mathbf{P}^{\cdot / h}\left(T_{A}^{-}<\infty\right) m$-q.e.

Proof First note that on $\left\{Y_{0} \in E\right\}, \quad\left\{S_{A}>0\right\}=\left\{S_{A} \circ \theta_{0}>0\right\}=\left\{T_{A}^{-} \circ \theta_{0}<\infty\right\}$. Therefore if $f \in p \mathcal{E}$

$$
\begin{aligned}
\widehat{R}_{A}(h \cdot m)(f) & =\mathbf{Q}_{m}^{h}\left[f \circ Y_{0} ; S_{A}>0\right] \\
& =\mathbf{Q}_{m}^{h}\left[f \circ Y_{0} \mathbf{P}^{Y(0) / h}\left(T_{A}^{-}<\infty\right)\right]=m\left[h f \mathbf{P}^{/ h}\left(T_{A}^{-}<\infty\right)\right] .
\end{aligned}
$$

But $\widehat{R}_{A}(h \cdot m)(f)=m\left(f p_{A} h\right)$ and since this holds for all $f \in p \mathcal{E}, \quad p_{A} h=$ $h \mathbf{P}^{\cdot / h}\left(T_{A}^{-}<\infty\right), m$-a.e. One checks easily that $\mathbf{P}^{\cdot / h}\left(T_{A}^{-}<\infty\right)$ is $h$-excessive and so there exists a unique excessive function $u_{A}$ with $u_{A}=h \mathbf{P} / h\left(T_{A}^{-}<\infty\right)$ on $\{h<\infty\}$. [3, Prop. 11.7]. Now $h<\infty, m$-a.e., hence $p_{A} h=u_{A}, m$-a.e., and then $m$-q.e.; in particular $p_{A} h=h \mathbf{P}^{/ h}\left(T_{A}^{-}<\infty\right) m$-q.e. since $\{h=\infty\}$ is $m$-polar.

Remark What has been proved so far for $S_{A}$ is also valid if we replace $Y_{t-}^{c r}$ in the definition of $S_{A}$ by $Y_{t-}^{r}$, which exists in $\bar{E}$, or if $Y$ has left limits in $E$ in the original topology by $Y_{t-}$. When comparing our results with those in Section 13.12 of [3], one should note that it is assumed there that $X$ and $\widehat{X}$ are in strong duality with respect to $m$, although branch points are allowed. Hence $X$ and $Y$ have left limits in the original topology. In particular if we use $Y_{t-}$ in the definition of $S_{A}$ it follows from Proposition 8.4 that $p_{A} h$ defined above agrees with $p_{A} h$ as defined in [3].

Recall the Ray compactification $\widetilde{E}$ and the Ray process $\widetilde{X}$ of Theorem 5.4. We change the notation slightly and let $\widetilde{X}$ denote the restriction of this Ray process to its set of non-branch points $\widetilde{D}$-this was denoted by $\widetilde{X}^{\widetilde{D}}$ in the last paragraph of Section 5. Then $\widetilde{X}$ is a Borel right process in the co-Ray topology, taking values in $\widetilde{D}$. In what follows it is important to note that (i) $E \backslash \widehat{B} \subset \widetilde{D}$, and (ii) if $\mu$ is a measure on $E \backslash \widehat{B}$ then the laws of $\left(\widehat{X}_{t}: t>0\right)$ under $\widehat{\mathbf{P}}^{\mu}$ and of $\left(\widetilde{X}_{t-}: t>0\right)$ under $\widetilde{\mathbf{P}}^{\mu}$ are the same. Of course, $\widetilde{X}_{t-}$ denotes the limit taken in the co-Ray topology. Since $m(\widehat{B})=0$, if $\xi \in \widehat{\operatorname{Exc}}(m)$ then $\xi \in \widetilde{\operatorname{Exc}}$, as was pointed out at the end of Section 5.

Although $\widehat{\operatorname{Pot}}(m)$ need not be a solid subcone of $\widehat{\operatorname{Exc}}(m)$, the next result shows that $\widehat{R}_{A} \xi \in \widehat{\operatorname{Pot}}(m)$ if $\xi=h \cdot m \in \widehat{\operatorname{Pot}}(m)$, under a mild condition on $A$. This generalizes [3, Prop. 13.62]. To state this result we need to introduce some notation. If $A \in \mathcal{E}$ with $A \subset E \backslash \widehat{B}$, define $\widetilde{A}$ to be the fine closure of $A$ in $\widetilde{D}$ relative to the Borel right process $\widetilde{X}$. For $t \geq 0$ define $\widehat{X}_{t}^{+}:=\widehat{X}_{t+}^{c r}$, which exists in $\widetilde{D}, \widehat{\mathbf{P}}^{x}$-a.s. for $x \in E \backslash \widehat{B}$. Note that $\left(\widehat{X}_{t}^{+}: t \geq 0\right)$ under $\widehat{\mathbf{P}}^{\mu}$ has the same law as $\left(\tilde{X}_{t}: t \geq 0\right)$ under $\widetilde{\mathbf{P}}^{\mu}$ provided $\mu$ is carried by $E \backslash \widehat{B}$. Moreover, for such $\mu$, if $\widetilde{D}_{A}:=\inf \left\{t>0: \widetilde{X}_{t} \in A\right\}$ and $\widehat{D}_{A}^{+}:=$ $\inf \left\{t>0: \widehat{X}_{t}^{+} \in A\right\}$, then the joint law of $\left(\widetilde{D}_{A}^{+},\left(\widetilde{X}_{t}: t \geq 0\right)\right)$ under $\widetilde{\mathbf{P}}^{\mu}$ is the same as that of $\left(\widehat{D}_{A}^{+},\left(\widehat{X}_{t}^{+}: t \geq 0\right)\right)$ under $\widehat{\mathbf{P}}^{\mu}$. These facts will be used freely in the remainder of this section. 
Proposition 8.5 Suppose $\xi=h \cdot m=\mu \widehat{U} \in \widehat{\operatorname{Pot}}(m)$ and $A \in \mathcal{E}$ with $\widetilde{A} \subset E \backslash \widehat{B}$. Then $\widehat{R}_{A} \xi=\mu_{A} \widehat{U} \in \widehat{\operatorname{Pot}}(m)$, where

$$
\mu_{A}=\mu \widetilde{P}_{\widetilde{D}_{A}}:=\widetilde{\mathbf{P}}^{\mu}\left(\widetilde{X}_{\widetilde{D}_{A}} \in \cdot\right)=\widehat{\mathbf{P}}^{\mu}\left(\widehat{X}_{\widehat{D}_{A}^{+}}^{+} \in \cdot\right) .
$$

Moreover, $\mu_{A}$ is carried by the union of $A$ and the points that are regular for $A$ with respect to $\widetilde{X}$. In particular, $\mu_{A}$ is carried by $\widetilde{A}$.

Proof Since $\mu$ is carried by $E \backslash \widehat{B}$, the assertions in the last sentence of Proposition 8.5 are clear; in particular, $\mu_{A}$ is carried by $E \backslash \widehat{B}$. Since the resolvent kernels $\widehat{U}^{q}(x, \cdot)$ and $\widetilde{U}^{q}(x, \cdot)$ are equal for $q>0$ and $x \in E \backslash \widehat{B}$ and $E$ is Lusin, it follows that $\left\{t: \widehat{P}_{t}(x, \cdot) \neq \widetilde{P}_{t}(x, \cdot)\right\}$ is at most countable for each $x \in E \backslash \widehat{B}$. Therefore for $f \in p \mathcal{E}$,

$$
\mu_{A} \widehat{U}(f)=\lim _{n} \int_{1 / n}^{\infty} \mu_{A} \widehat{P}_{t}(f) d t=\lim _{n} \int_{0}^{\infty} \mu_{A} \widetilde{P}_{t+1 / n}(f) d t .
$$

Using the strong Markov property of $\widetilde{X}$ under $\widetilde{\mathbf{P}}^{\mu}$ for the second equality,

$$
\mu_{A} \widetilde{P}_{t+1 / n}(f)=\widetilde{\mathbf{P}}^{\mu}\left[\widetilde{P}_{t+1 / n} f \circ \widetilde{X}_{\widetilde{D}_{A}}\right]=\widetilde{\mathbf{P}}^{\mu}\left[f \circ \widetilde{X}_{\widetilde{D}_{A}+t+1 / n}\right],
$$

and consequently

$$
\begin{aligned}
\mu_{A} \widehat{U}(f) & =\lim _{n} \widetilde{\mathbf{P}}^{\mu} \int_{\widetilde{D}_{A}+1 / n}^{\infty} f \circ \widetilde{X}_{t} d t \\
& =\widetilde{\mathbf{P}}^{\mu} \int_{\widetilde{D}_{A}}^{\infty} f \circ \widetilde{X}_{t} d t=\widehat{\mathbf{P}}^{\mu} \int_{\widehat{D}_{A}^{+}}^{\infty} f \circ \widehat{X}_{t} d t .
\end{aligned}
$$

Since $h \cdot m \in \widehat{\operatorname{Pot}}(m), h \in \mathcal{S}_{p}(m)$ and $\widehat{v}_{t}:=\mu \widehat{P}_{t}$ is the unique loose entrance law with $\mu \widehat{U}=\int_{0}^{\infty} \widehat{v}_{t} d t$. Thus from Theorem $6.4, \mathbf{Q}_{m}^{h}=\int \sigma_{t} \widehat{\mathbf{Q}}_{\nu} d t$. But $\mu$ is carried by $E \backslash \widehat{B}$, and so $\left(\widehat{X}_{t}: t \geq 0\right)$ has the same law under $\widehat{\mathbf{P}}^{\mu}$ and $\widehat{\mathbf{Q}}_{v}$ when $\widehat{X}_{0}:=\widehat{X}_{0+}^{c r}$ according to Remark 7.5. This implies that the joint law of $\left(\widehat{X}_{t}, \widehat{D}_{A}^{+}\right)$is the same under $\widehat{\mathbf{P}}^{\mu}$ and $\widehat{\mathbf{Q}}_{\nu}$. Therefore $\mu_{A} \widehat{U}(f)=\widehat{\mathbf{Q}}_{v} \int_{\widehat{D}_{A}^{+}}^{\infty} f \circ \widehat{X}_{t} d t$. But $\widehat{\mathbf{Q}}_{v}(\beta \neq 0)=0$ and so $\widehat{\mathbf{Q}}_{v}$-a.e.,

$$
\widehat{D}_{A}^{+}=\inf \left\{t \geq 0: \widehat{X}_{t}^{+} \in A\right\}=-\sup \left\{t \leq 0 ; Y_{t-}^{c r} \in A\right\}=-S_{A} .
$$

Hence

$$
\begin{aligned}
\mu_{A} \widehat{U}(f) & =\widehat{\mathbf{Q}}_{v} \int_{-\infty}^{S_{A}} f \circ Y_{t} d t=\int_{-\infty}^{\infty} \widehat{\mathbf{Q}}_{v}\left[f \circ Y_{t} ; S_{A}>t\right] d t \\
& =\mathbf{Q}_{m}^{h}\left[f \circ Y_{0} ; S_{A}>0\right]=\widehat{R}_{A}(h \cdot m)(f),
\end{aligned}
$$

because $S_{A}-t=S_{A} \circ \sigma_{t}$. As noted before $\mu_{A}$ is carried by $\widetilde{A} \subset E \backslash \widehat{B}$ and so $\widehat{R}_{A}(h \cdot m) \in \widehat{\operatorname{Pot}}(m)$.

Proposition 8.5 describes $\widehat{R}_{A} \xi$ when $\xi \in \widetilde{\widetilde{P}} \widetilde{\sim}(m)$. The next result describes $\widehat{R}_{A} \xi$ when $\xi \in \widehat{\operatorname{Har}}(m)$. In its statement, $\widetilde{L}$ denotes the energy functional of the Borel right process $\widetilde{X}$.

Proposition 8.6 Let $A \in \mathcal{E}$ with $A \subset E \backslash \widehat{B}$ and $\xi=h \cdot m$, where $h \in \mathcal{S}(m)$. 
(i) If $m \in$ Con, let $\phi:=\mathbf{P}^{\bullet}\left(T_{A}^{-}<\infty\right)$. Then $\phi(x)$ is either 0 or 1 for $m$-a.e. $x$, and $p_{A} h=\phi \cdot h$.

(ii) If $m \in \mathrm{Dis}$ and $\xi=h \cdot m \in \widehat{\operatorname{Har}}(m)$, then

$$
\widehat{R}_{A} \xi(f)=\widetilde{L}\left(h \cdot m, \widetilde{P}_{A} \widetilde{U} f\right),
$$

where $\widetilde{P}_{A} g:=\widetilde{\mathbf{P}}^{\bullet}\left(g \circ \widetilde{X}_{\widetilde{T}(A)}\right)$ and $\widetilde{T}(A):=\inf \left\{t>0: \widetilde{X}_{t} \in A\right\}$.

\section{Proof}

(i) Fix $m \in$ Con. Theorem 2.16 in [13] asserts that $\mathbf{P}^{x}\left[h\left(X_{t}\right) \neq h\left(X_{0}\right)\right.$ for some $t>0]=0$ for $m$-a.e. $x$. In particular, $P_{t} h(x)=h(x)$ for all $t \geq 0$, for $m$-a.e. $x$. But this implies that $P_{t}^{h} 1=1, m$-a.e. Consequently, $\mathbf{P}^{\bullet / h}(\zeta=\infty)=1, m$-a.e. Therefore

$$
p_{A} h=h \cdot \mathbf{P}^{\bullet / h}\left(T_{A}^{-}<\infty\right)=\mathbf{P}^{\bullet}\left(h \circ X_{T_{A}^{-}} ; T_{A}^{-}<\infty\right)=h \phi .
$$

Set $T:=T_{A}^{-}$for notational convenience. Since $T$ is an exact terminal time, we have for $t>0$ and $m$-a.e. $x$,

$$
\begin{aligned}
\phi(x) & =\mathbf{P}^{x}(T<\infty)=\mathbf{P}^{x}(T \leq t)+\mathbf{P}^{x}(t<T<\infty) \\
& =\mathbf{P}^{x}(T \leq t)+\mathbf{P}^{x}\left(\phi\left(X_{t}\right) ; t<T\right) .
\end{aligned}
$$

Let $t \rightarrow \infty$ above and use the fact that $\phi$ is excessive (so that $\mathbf{P}^{x}\left[\phi\left(X_{t}\right) \neq \phi\left(X_{0}\right)\right.$ for some $t>0]=0$ for $m$-a.e. $x)$ to obtain $\phi=\phi+\phi(1-\phi)$, which proves (i).

(ii) For each $x \in E_{\Delta}$, Theorem 5.4 implies that $\left(\widehat{X}_{t}: t>0\right)$ under $\widehat{\mathbf{P}}^{x}$ and $\left(\widetilde{X}_{t-}\right.$ : $t>0$ ) under $\widetilde{\mathbf{P}}^{x}$ have that same law. Of course limits involving $\tilde{X}$ refer to the co-Ray topology of $\widetilde{E}$. If, as in Section $7, \widehat{X}_{0}:=\widehat{X}_{0+}^{c r}$ and also $\widetilde{X}_{0-}:=\widetilde{X}_{0}$, then this assertion extends to $t=0$. It was pointed out at the end of Section 5 that $\xi \in \widetilde{\text { Exc. Let }} \widetilde{\mathbf{Q}}_{\xi}$ and $\widetilde{Y}$ be the Kuznetsov measure and process corresponding to $\xi$ and the semigroup $\left(\widetilde{P}_{t}\right)$ of $\widetilde{X}$. Thus $\left(\widetilde{Y}_{t}\right)$ is right continuous with values in $\widetilde{D}$ and left limits $\widetilde{Y}_{t-}$ in $\widetilde{E}$ for $\widetilde{\alpha}<t<\widetilde{\beta}$. Topological statements about $\widetilde{Y}$ refer to the co-Ray topology of $\widetilde{E}$. Since $\xi$ is carried by $E$, in fact by $E \backslash \widehat{B},\left(Y_{-t}^{\#}\right)$ under $\mathbf{Q}_{m}^{h}$ has the same law as $\left(\widetilde{Y}_{t-}^{\#}\right)$ under $\widetilde{\mathbf{Q}}_{\xi}$. But $\xi=h \cdot m \in \widetilde{\operatorname{Har}}(m)$ and so $Y_{-t}^{\#}$ is equivalent to $Y_{-t}^{*}$ under $\mathbf{Q}_{m}^{h}$, which has the same law as $\widetilde{Y}_{t-}^{*}=\widetilde{Y}_{t-}$ under $\widetilde{\mathbf{Q}}_{\xi}$. Also

$$
S_{A}=\sup \left\{t>\alpha: Y_{t-}^{c r} \in A\right\}=-\inf \left\{t<-\alpha: Y_{(-t)-}^{c r} \in A\right\} .
$$

Hence $S_{A}$ under $\mathbf{Q}_{m}^{h}$ and $-\widetilde{\tau}_{A}$ under $\widehat{\mathbf{Q}}_{\xi}$ have the same law, where $\widetilde{\tau}_{A}:=\inf \{t$ : $\left.\widetilde{Y}_{t} \in A\right\}$ because the processes $\left(Y_{(-t)-}^{c r}, \mathbf{Q}_{m}^{h}\right)$ and $\left(\widetilde{Y}_{t}, \widetilde{\mathbf{Q}}_{\xi}\right)$ are identical in law. But for each $t, \mathbf{Q}_{m}^{h}\left(Y_{t-}^{c r} \neq Y_{t}\right)=0$. Consequently,

$$
\begin{aligned}
\widehat{R}_{A}(h \cdot m)(f) & =\mathbf{Q}_{m}^{h}\left(f \circ Y_{0} ; S_{A}>0\right) \\
& =\mathbf{Q}_{m}^{h}\left(f \circ Y_{0-}^{c r} ; S_{A}>0\right) \\
& =\widetilde{\mathbf{Q}}_{\xi}\left(f \circ \widetilde{Y}_{0} ; \widetilde{\tau}_{A}<0\right)=\widetilde{R}_{A} \xi(f),
\end{aligned}
$$

where $\widetilde{R}_{A} \xi$ is the balayage of $\xi$ on $A$ relative to the $\operatorname{Kuznetsov}$ process $\left(\widetilde{Y}, \widetilde{\mathbf{Q}}_{\xi}\right)$; see $[13,(7.3),(7.9)]$. 
Since $m \in$ Dis, there exists an increasing sequence $\left(U f_{n}\right)$ of potentials with $U f_{n} \uparrow h, m$-a.e. Define $\eta_{n}:=f_{n} m$. Then $\eta_{n}$ is carried by $E \backslash \widehat{B} \subset \widetilde{D}$ and

$$
\eta_{n} \widetilde{U} f=\eta_{n} \widehat{U} f=\left(f_{n}, \widehat{U} f\right)=\left(U f_{n}, f\right) \uparrow m(h f)=\xi(f) .
$$



Remark The proof of Proposition 8.6(ii) does not require $m \in$ Dis. It suffices that $h \cdot m \in \widetilde{\operatorname{Har}}(m)$ and that there exists an increasing sequence $\left(\eta_{n} \widehat{U}\right) \subset \widehat{\operatorname{Pot}}(m)$ with $\eta_{n} \widehat{U} \uparrow h \cdot m$.

Propositions 8.5 and 8.6 combine to yield the following description of $\widehat{R}_{A} \xi$ when $m$ is dissipative.

Theorem 8.7 Let $A \in \mathcal{E}$ with $\widetilde{A} \subset E \backslash \widehat{B}, m \in \operatorname{Dis}$, and $h \in \mathcal{S}(m)$. Let $\mu \widehat{U}+\eta$ be the Riesz decomposition of $\xi:=h \cdot m$ into its co-potential and co-harmonic parts. Then, for $f \in p \mathcal{E}$,

$$
\widehat{R}_{A} \xi(f)=\mu \widetilde{P}_{\widetilde{D}(A)} \widehat{U} f+\widetilde{L}\left(\eta, \widetilde{P}_{A} \widetilde{U} f\right)=\sup \left\{v \widetilde{P}_{\widetilde{D}(A)} \widehat{U} f: v \widetilde{U} \leq \xi\right\} .
$$

Proof The first equality in Eq. 8.5 is an immediate corollary of Propositions 8.5 and 8.6. For the second let $u:=\widetilde{P}_{\widetilde{D}(A)} \widehat{U} f=\widetilde{P}_{\widetilde{D}(A)} \widetilde{U} f$, since $\widetilde{A} \subset E \backslash \widehat{B}$. Now $\widetilde{D}(A)$ is an exact terminal time for $\widetilde{X}$ whose exact regularization is $\widetilde{T}_{A}$. It is easy to check that $u$ is strongly supermedian with excessive regularization $\bar{u}:=\widetilde{P}_{A} \widetilde{U} f$; that is, $\widetilde{P}_{t} u \uparrow \bar{u}$ as $t \downarrow 0$. The second equality in Eq. 8.5 now follows from Theorem (4.7) of [11].

We next list some properties of the map $\xi \mapsto{\underset{\sim}{R}}_{A} \xi$ from $\widehat{\operatorname{Exc}}(m)$ to $\widehat{\operatorname{Exc}}(m)$, for $A \in \mathcal{E}$. Recall that if $A \in \mathcal{E}$ with $A \subset E \backslash \widehat{B}$ then $\widetilde{A}$ denotes the fine closure of $A$ in $\widetilde{D}$ relative to $\widetilde{X}$. It was noted earlier that $\widehat{R}_{A} \xi \leq \xi$ and $\widehat{R}_{A}(\xi+\eta)=\widehat{R}_{A} \xi+\widehat{R}_{A} \eta$ for $\xi, \eta \in \widehat{\operatorname{Exc}}(m)$.

Proposition 8.8 Let $m \in$ Dis, $A \in \mathcal{E}$ with $\widetilde{A} \subset E \backslash \widehat{B}$.

(i) If $\xi, \eta \in \widehat{\operatorname{Exc}}(m)$ with $\xi \leq \eta$, then $\widehat{R}_{A} \xi \leq \widehat{R}_{A} \eta$.

(ii) If $\xi \in \widehat{\operatorname{Har}}(m)$, then there is a sequence $\left(\mu_{n} \widehat{U}\right) \subset \widehat{\operatorname{Pot}}(m)$ with $\mu_{n} \widehat{U} \uparrow \xi$, and for any such sequence we have $\widehat{R}_{A}\left(\mu_{n} \widehat{U}\right) \uparrow \widehat{R}_{A} \xi$.

(iii) For any $m \in \operatorname{Exc}, \xi \in \widehat{\operatorname{Exc}}(m)$, and $A, B \in \mathcal{E}$ with $A \subset B$, we have $\widehat{R}_{A} \widehat{R}_{B} \xi=$ $\widehat{R}_{B} \widehat{R}_{A} \xi=\widehat{R}_{A} \xi \leq \widehat{R}_{B} \xi$

Proof Assertion (i) is an immediate consequence of the second expression for $\widehat{R}_{A} \xi$ in Eq. 8.5. For (ii), as in the last paragraph of the proof of Proposition 8.6, there exists a sequence $\left(\mu_{n} \widehat{U}\right) \subset \widehat{\operatorname{Pot}}(m)$ with $\mu_{n} \widehat{U} \uparrow \xi$. If $\left(\mu_{n} \widehat{U}\right)$ is any such sequence, it follows from (i) that $\widehat{R}_{A}\left(\mu_{n} \widehat{U}\right)$ increases and $\lim _{n} \widehat{R}_{A}\left(\mu_{n} \widehat{U}\right) \leq \widehat{R}_{A} \xi$. If $f \in p \mathcal{E}$, then $\widehat{R}_{A}\left(\mu_{n} \widehat{U}\right)(f)=\mu_{n}(u)$, with $u=\widetilde{P}_{\widetilde{D}(A)} \widetilde{U} f$, according to Propositions 8.5. Now $\mu_{n}(u) \uparrow a \leq \widehat{R}_{A} \xi(f)=\widetilde{L}(\xi, \bar{u})$ where $\bar{u}=\widetilde{P}_{A} \widetilde{U} f$ is the $\widetilde{X}$-excessive regularization of $u$ as before. But $\widetilde{L}(\xi, \bar{u})=\uparrow \lim _{n} \mu_{n}(\bar{u})$ because $\mu_{n} \widetilde{U}=\mu_{n} \widehat{U} \uparrow \xi$. However $\bar{u} \leq u$, so $\mu_{n}(u) \geq \mu_{n}(\bar{u})$ which forces $a \geq \widetilde{L}(\xi, \bar{u})$, establishing (ii). For (iii), $A \subset B$ implies $S_{A} \leq S_{B}$ and so from the definition (8.1), $\widehat{R}_{A} \xi \leq \widehat{R}_{B} \xi$. Also, $k_{S_{A}} \circ k_{S_{B}}=k_{S_{B}} \circ k_{S_{A}}=$ $k_{S_{A}}$. Hence the remaining assertions in (iii) follow from Proposition 8.2. 
The next result is a direct generalization of Theorem 13.65 in [3]. For its statement we need some notation. If $\mu$ is a measure on $E$, let $\operatorname{Supp}(\mu, c r)$ denote the co-Ray support of $\mu$ and for $A \in \mathcal{E}$, define $M(A)$ to be the set of measures $\mu$ on $E$ such that $\operatorname{Supp}(\mu, c r)$ is compact (in the co-Ray topology) and contained in $A$.

Theorem 8.9 Suppose $m \in \mathrm{Dis}, h \cdot m \in \widehat{\operatorname{Exc}}(m), A \in \mathcal{E}$ and $A \subset E \backslash \widehat{B}$. Then

$$
\widehat{R}_{A}(h \cdot m)=\sup \{\mu \widehat{U} \in \widehat{\operatorname{Pot}}(m): \mu \widehat{U} \leq h \cdot m, \mu \in M(A)\} .
$$

Proof Suppose $\mu \widehat{U} \in \widehat{\operatorname{Pot}}(m), \mu \widehat{U} \leq h \cdot m$ and $\mu \in M(A)$. Let $K=\operatorname{Supp}(\mu, c r) \subset$ $A \subset E \backslash \widehat{B}$. Then from Propositions 8.8 and 8.5 ,

$$
\widehat{R}_{A}(h \cdot m) \geq \widehat{R}_{A}(\mu \widehat{U}) \geq \widehat{R}_{K}(\mu \widehat{U})=\mu_{K} \widehat{U}
$$

where $\mu_{K}=\mu \widetilde{P}_{\widetilde{D}_{(K)}}$. But $K$ is co-Ray compact and carries $\mu$. Therefore $\mu_{K}=\mu$ and $\mu \widehat{U}=\mu_{K} \widehat{U} \leq \widehat{R}_{A}(h \cdot m)$. Thus $\widehat{R}_{A}(h \cdot m)$ dominates the supremum in Eq. 8.6. For the reverse inequality, we shall first show that there exists an increasing sequence $\left(K_{n}\right)$ of co-Ray compact subsets of $A$ with $\widehat{R}_{K_{n}}(h \cdot m) \uparrow \widehat{R}_{A}(h \cdot m)$. A theorem of Dellacherie (see $[12,(12.15)])$ implies that there is an increasing sequence $\left(K_{n}\right)$ of coRay compact subsets of $A$ with $\widetilde{T}_{K_{n}} \downarrow \widetilde{T}_{A}, \widetilde{\mathbf{P}}^{\xi}$-a.s., where $\xi=h \cdot m$ as usual. It follows that $\tilde{\tau}_{K_{n}} \downarrow \widetilde{\tau}_{A}, \widetilde{\mathbf{Q}}_{\xi}$-a.e., where the notation is as in the proof of Proposition 8.6(ii). Thus, by Eq. 8.4,

$$
\begin{aligned}
\widehat{R}_{K_{n}} \xi(f) & =\widetilde{\mathbf{Q}}_{\xi}\left[f \circ \tilde{Y}_{0} ; \tilde{\tau}_{K_{n}}<0\right] \\
& \uparrow \widetilde{\mathbf{Q}}_{\xi}\left[f \circ \widetilde{Y}_{0} ; \tilde{\tau}_{A}<0\right]=\widehat{R}_{A} \xi(f) .
\end{aligned}
$$

Now let $\rho \widehat{U}+\eta$ be the decomposition of $\xi$ into its co-potential part $\rho \widehat{U}$ and its co-harmonic part $\eta$. From Proposition 8.8 there exists a sequence $\left(\mu_{k} \widehat{U}\right) \subset \widehat{\operatorname{Pot}}(m)$ increasing to $\eta$ with $\widehat{R}_{K_{n}}\left(\mu_{k} \widehat{U}\right) \uparrow \widehat{R}_{K_{n}} \eta$. Let $\lambda_{k}:=\rho+\mu_{k}$. Then $\widehat{R}_{K_{n}}\left(\lambda_{k} \widehat{U}\right)=\mu_{n, k} \widehat{U}$, where $\mu_{n, k}:=\lambda_{k} \widetilde{P}_{\widetilde{D}\left(K_{n}\right)}$ and $\operatorname{supp}\left(\mu_{n, k}, c r\right) \subset K_{n} \subset A$. Hence $\mu_{n, k} \in M(A)$. Now $\mu_{n . k} \widehat{U}=\widehat{R}_{K_{n}}(\rho \widehat{U})+\widehat{R}_{K_{n}}\left(\mu_{k} \widehat{U}\right)$ increases with both $n$ and $k$, and

$$
\lim _{n} \lim _{k} \mu_{n, k} \widehat{U}=\lim _{n} \widehat{R}_{K_{n}} \xi=\widehat{R}_{A} \xi \text {. }
$$

Therefore $\mu_{n, n} \in M(A)$, and $\mu_{n, n} \widehat{U} \uparrow \widehat{R}_{A} \xi$, completing the proof of Theorem 8.9.

Concluding Remarks In comparing the results in this section to those in Section 13.12 of [3], one should note that it is assumed there that $X$ and $\widehat{X}$ are right continuous strong Markov process in strong duality although they allow both $X$ and $\widehat{X}$ to have branch points. Moreover most of their deeper results such as $13.59,13.62$ and 13.65 depend on the hypothesis $(\widehat{S F})$ on page 371 . This is not made explicit but their proofs use 13.50. Under $(\widehat{S F})$ one may use the original topology in defining the co-Ray compactification $\widetilde{E}$ as remarked at the end of the first paragraph of Section 5. But then (15.1) and (15.3) of [12] imply that the co-Ray topology agrees with the original topology. Therefore those results are corollaries of the results in this section.

It seems that in 13.65 of [3] it is necessary to suppose that $A \subset E \backslash \widehat{B}$.

Open Access This article is distributed under the terms of the Creative Commons Attribution Noncommercial License which permits any noncommercial use, distribution, and reproduction in any medium, provided the original author(s) and source are credited. 


\section{References}

1. Blumenthal, R.M., Getoor, R.K.: Markov Processes and Potential Theory. Academic, New York (1968)

2. Chung, K.L.: A Course in Probability Theory, 3rd edn. Academic, New York (2001)

3. Chung, K.L., Walsh, J.B.: Markov Processes, Brownian Motion, and Time Symmetry, 2nd edn. Springer, New York (2005)

4. Dellacherie, C., Meyer, P.-A.: Probabilités et Potentiel. Chapitres I à IV. Hermann, Paris (1975)

5. Dellacherie, C., Meyer, P.-A.: Probabilités et Potentiel. Chapitres V à VIII. Théorie des martingales. Hermann, Paris (1980)

6. Dellacherie, C., and Meyer, P.-A.: Probabilités et Potentiel. Chapitres XII-XVI. Théorie du potentiel associée à une résolvante. Théorie des processus de Markov. Hermann, Paris (1987)

7. Dellacherie, C., Meyer, P.-A., Maisonneuve, B.: Probabilités et Potentiel. Chapitres XVIIXXIV. Processus de Markov (fin), Compléments de calcul stochastique. Hermann, Paris (1992)

8. Fitzsimmons, P.J., Maisonneuve, B.: Excessive measures and Markov processes with random birth and death. Probab. Theory Relat. Fields 72, 319-336 (1986)

9. Fitzsimmons, P.J.: Homogeneous random measures and a weak order for the excessive measures of a Markov process. Trans. Am. Math. Soc. 303 (1987) 431-478.

10. Fitzsimmons, P.J., Getoor, R.K.: A fine domination principle for excessive measures. Math. Z. 207, 137-151 (1991)

11. Fitzsimmons, P.J., Getoor, R.K.: Homogeneous random measures and strongly supermedian kernels of a Markov process. Electron. J. Probab. 8(10), 55 (2003)

12. Getoor, R.K.: Markov Processes: Ray Processes and Right Processes. Lecture Notes in Mathematics, vol. 440. Springer, Berlin (1975)

13. Getoor, R.K.: Excessive Measures. Birkhäuser, Boston (1990)

14. Getoor, R.K.: Measure perturbations of Markovian semigroups. Potential Anal. 11, 101-133 (1999)

15. Getoor, R.K., Glover, J.: Riesz decompositions in Markov process theory. Trans. Am. Math. Soc. 285, 107-132 (1984)

16. Getoor, R.K., Sharpe, M.J.: Naturality, standardness, and weak duality for Markov processes. Z. Wahrscheinlichkeitstheor. Verw. Geb. 67, 1-62 (1984)

17. Janssen, K.: Representation of excessive measures. In: Seminar on Stochastic Processes, 1986, pp. 85-105. Birkhäuser, Boston (1987)

18. Kuznetsov, S.E.: Construction of Markov processes with random times of birth and death. Theory Probab. Appl. 18, 571-575 (1973)

19. Sharpe, M.: General Theory of Markov Processes. Academic, Boston (1988)

20. Walsh, J.B.: Two footnotes to a theorem of Ray. In: Séminaire de Probabilités, V. Lecture Notes in Math, 191, pp. 283-289. Springer, Berlin (1971) 